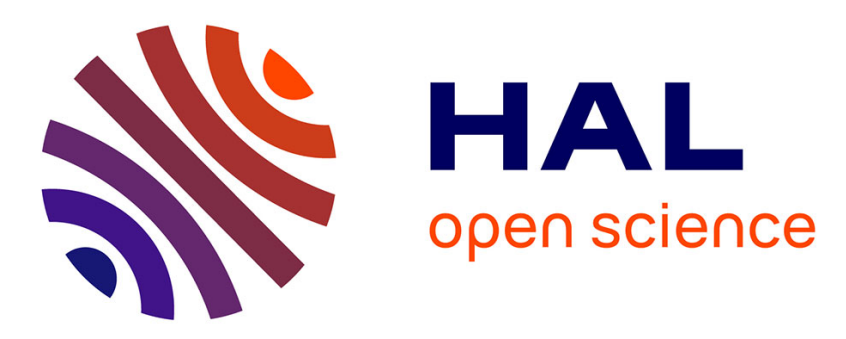

\title{
The interaction of thermal phonons with conduction electrons in InSb. Application to heat-pulse spectroscopy
}

D. Huet, B. Pannetier, F.R. Ladan, J.P. Maneval

\section{- To cite this version:}

D. Huet, B. Pannetier, F.R. Ladan, J.P. Maneval. The interaction of thermal phonons with conduction electrons in InSb. Application to heat-pulse spectroscopy. Journal de Physique, 1976, 37 (5), pp.521536. 10.1051/jphys:01976003705052100 . jpa-00208447

\section{HAL Id: jpa-00208447 https://hal.science/jpa-00208447}

Submitted on 1 Jan 1976

HAL is a multi-disciplinary open access archive for the deposit and dissemination of scientific research documents, whether they are published or not. The documents may come from teaching and research institutions in France or abroad, or from public or private research centers.
L'archive ouverte pluridisciplinaire HAL, est destinée au dépôt et à la diffusion de documents scientifiques de niveau recherche, publiés ou non, émanant des établissements d'enseignement et de recherche français ou étrangers, des laboratoires publics ou privés. 
Classification

Physics Abstracts

7.660

\title{
THE INTERACTION OF THERMAL PHONONS WITH CONDUCTION ELECTRONS IN InSb. APPLICATION TO HEAT-PULSE SPECTROSCOPY
}

\author{
D. HUET, B. PANNETIER, F. R. LADAN and J. P. MANEVAL \\ Groupe de Physique des Solides de l'Ecole Normale Supérieure et Université Paris VII, \\ 24, rue Lhomond, 75231 Paris 05, France
}

(Reçu le 22 septembre 1975, accepté le 5 janvier 1976)

\begin{abstract}
Résumé. - Des phonons balistiques de haute fréquence ont été émis dans l'antimoniure d'indium dégénéré de type $\mathrm{n}$ par la méthode des impulsions de chaleur $\left(T_{0}<4,2 \mathrm{~K}\right)$. Nous passons d'abord en revue les aspects correspondants de l'interaction électron-phonon : la coupure pour les vecteurs d'onde de phonons excédant le double du rayon de Fermi $\left(q>2 k_{\mathrm{F}}\right)$, le couplage préférentiel des modes longitudinaux, et l'effet d'écran.

Puis nous rendons compte de nouvelles mesures approfondies sur l'absorption des phonons en fonction de la concentration électronique dans le semiconducteur. La sphère de Fermi se comporte comme un filtre passe-haut et permet une spectroscopie des phonons sans paramètre ajustable. Ainsi la distribution en fréquences d'une impulsion de chaleur a-t-elle pu être déterminée de façon détaillée.

Abstract. - High frequency ballistic phonons were radiated by the heat pulse method $\left(T_{0}<4.2 \mathrm{~K}\right)$ into degenerate n-type indium antimonide crystals. We first review the relevant aspects of the electronphonon interaction : cut-off for phonon wavevectors larger than twice the Fermi radius $\left(q>2 k_{\mathrm{F}}\right)$, selective coupling of the longitudinal modes and screening effects.

Then, we report original extensive measurements of phonon absorption as a function of the electron concentration in the semiconductor. The Fermi sphere acts as a high-pass filter, which enables phonon spectroscopy with no adjustable parameters. As a result, the frequency distribution of a heat pulse could be determined in a detailed fashion.
\end{abstract}

1. Introduction. - The transport properties of phonons at low temperatures are by now being studied by the ballistic heat-pulse method [1,2] concurrently with the more developed steady-state heat conductivity method. Attractive features of the former are the possibilities of selecting the propagation and polarization directions of the phonons, and of generating far from equilibrium distributions. Thus, it has become possible to investigate in more detail than before the interactions of excess phonons with other excitations in crystals including impurity levels, electrons, equilibrium phonons, etc... In particular, heat pulse attenuation measurements [3] led to a direct check of the selection rules for the deformation potential in a semiconductor. The electron-phonon interaction is here developed into a spectroscopic analyzer of high-frequency $\left(10^{10}\right.$ to $\left.10^{12} \mathrm{~Hz}\right)$ phonons.

One considers in this work ballistic phonons of rather short wavelengths (typically $100 \AA$ ) interacting with a degenerate Fermi gas of electrons. In the quantum absorption process, the phonon wave- vector $q$ must be smaller than twice the radius $k_{\mathrm{F}}$ of the Fermi sphere :

$$
q \lesssim 2 k_{\mathrm{F}}
$$

a condition implied by the conservation of quasimomentum and of energy. It was verified (for emission processes) by X-ray analysis of piezoelectrically amplified acoustic flux [4] in GaAs, and (for absorption) by heat-pulse attenuation measurements [5] on doped InSb and $\mathrm{GaSb}$. The principle of our spectroscopy follows : let a given phonon beam of spectral power density $P(q)$ be transmitted through a slab of a degenerate semiconductor. Up to $q=2 k_{\mathrm{F}}$ the phonons are absorbed by the electrons and substracted from the ballistic beam. By selecting samples of increasing carrier concentration, one gradually scans the whole phonon spectrum. This enables the derivation of the distribution $P(q)$ without any assumption concerning its shape. In this respect, the $2 k_{\mathrm{F}}$ spectroscopy is essentially different than the earlier heat pulse analysis $[6,7]$, which both relied on 
an a priori form of the spectrum (e.g. the Planck radiation model), and yielded the power dependence of the mean phonon frequency rather than the true spectrum at a given emission power. On the other hand, the X-ray probe [4] or the spin-phonon spectrometer [8] which were also used to measure phonon distributions, are not readily applicable to transient analysis.

The most commonly used generators of highfrequency phonons are metallic films driven by electrical (or laser) pulses [1]. These incoherent broadband sources are referred to as Joule emitters. Although they are surpassed in many respects by the newly developed quasi-monochromatic sources they were studied first for convenience.

We performed detailed measurements of the heatpulse attenuation in indium antimonide crystals representing a wide variety of dopings $\left(10^{14}\right.$ to $10^{19}$ electrons per $\mathrm{cm}^{3}$ ). Section 2 outlines the elementary theory of the electron-phonon interaction. Heat pulse methods are described in section 3, with emphasis on the generation problem, while section 4 deals with special topics associated with crystalline anisotropy. Fundamental tests of the electron-phonon theory $[3,5]$ are reviewed in section 5 . Then, (section 6), the new attenuation experiments are presented. Attention is drawn to the care needed for obtaining significant quantitative results. Finally, the Joule emission spectra derived by means of the $2 k_{\mathrm{F}}$ spectroscopy are discussed and compared to the presently available theories of phonon emission (section 7). An application to thin-film resonances is also proposed.

2. The interaction of conduction electrons with acoustical phonons. - The electron-phonon interaction features a wide variety of behaviours according to the electron concentration (from the Maxwellian to the degenerate case) and the frequency of the phonons involved. Fortunately, the situation we are dealing with lends itself to a simple, though accurate enough, picture. On the one hand, thermal phonons at liquid helium temperatures still belong to the domain of long waves. On the other hand, they have high enough energies that both criteria $q l \gg 1$ and $\omega \tau>1$, where $l$ and $\tau$ are the electronic mean free path and lifetime respectively and $\omega$ the phonon angular frequency, are fulfilled, so that the quantum treatment of the interaction is adequate.

2.1 Matrix elements. - The conduction band of indium antimonide [9] is centered at $\mathbf{k}=\mathbf{0}$ and isotropic. The Bloch functions

$$
\psi_{\mathbf{k}}^{ \pm}(\mathbf{r})=U_{\mathbf{k}}^{ \pm}(\mathbf{r}) \exp (i \mathbf{k} \cdot \mathbf{r})
$$

are characterized by S-symmetry in the cell-periodic functions $U_{\mathbf{k}}^{ \pm}$and degeneracy with respect to the spin index \pm , at least for electron energies $\varepsilon(k)$ much smaller than the gap $(0.23 \mathrm{eV})$.

One distinguishes two origins of the coupling between lattice deformations and electronic states in III-V semiconductors [10]. The piezoelectric force, which arises from the polarization of the lattice under strain, is most important for the interpretation of ultrasonic attenuation and amplification data. For the phonons of comparatively high energy to be considered here, we will be mainly concerned with the shortranged deformation potential [11], which is wavemechanical in nature. In a state of uniform strain (strain tensor : $S_{i j}$ ), both effects can be represented by real quantities linear in the strain, namely a piezoelectric polarization field :

$$
P_{j}(\text { piezo })=e_{j k l} S_{k l}
$$

and, due to the deformation potential, an energy shift in the conduction states :

$$
\delta \varepsilon(\text { def })=b_{i j} S_{i j}
$$

The significance of $b_{i j}$, whose dependence upon the electron state is generally negligible near the center of the Brillouin zone, is related [12] to the ground-state cell-function $U_{0}^{ \pm}(\mathbf{r})$. In the limit of slowly varying strains ( $q \ll$ reciprocal lattice parameter), one can use $[11,12]$ the operator $\delta \varepsilon(\mathbf{r})=b_{i j} S_{i j}(\mathbf{r})$, where $S_{i j}(\mathbf{r})$ is the local strain, as the perturbation Hamiltonian causing transitions between the electronic states. In the same manner, the piezo-field is given by $e_{j k l} S_{k l}(\mathbf{r})$.

We consider transitions in which one phonon of wavevector $\mathbf{q}$ and of unit polarization vector $\mathbf{u}$ is absorbed. The initial and final electron wavevectors, $\mathbf{k}$ and $\mathbf{k}^{\prime}$, must satisfy the interference condition :

$$
\mathbf{k}+\mathbf{q}=\mathbf{k}^{\prime}
$$

with neglect of Umklapp processes for small $\mathbf{k}$ and $\mathbf{q}$. Within the effective mass approximation, the matrix elements of the equivalent potential $V_{\text {piezo }}$ and $V_{\text {def }}$ take on simple forms, without explicit dependence upon $\mathbf{k}$ and $\mathbf{k}^{\prime}$. The case of piezoelectricity was worked out by Meijer and Polder [13] with the result :

$$
\begin{aligned}
& \left|\left\langle\mathbf{k}\left|V_{\text {piezo }}(\mathbf{q}, \mathbf{u})\right| \mathbf{k}+\mathbf{q}\right\rangle\right|^{2}= \\
& =\frac{32 \pi^{2} \hbar e^{2}\left(e_{14}\right)^{2}}{\varepsilon_{\mathrm{r}}^{2} \rho s} \times \frac{1}{q} \times \\
& \quad \times\left(\bar{q}_{x} \bar{q}_{y} \bar{u}_{z}+\bar{q}_{y} \bar{q}_{z} \bar{u}_{x}+\bar{q}_{z} \bar{q}_{x} \bar{u}_{y}\right)^{2}
\end{aligned}
$$

In this equation, $\varepsilon_{\mathrm{r}}$ is the relative permittivity, $\rho$ the mass density of the crystal, and $e_{14}$ is the reduced component, $P_{x} /\left(S_{y z}+S_{z y}\right)$, of the $e_{j k l}$ tensor for the zinc-blende structure. The $\bar{u}_{j}$ and $\bar{q}_{j}$ are respectively the direction cosines of the polarization vector $\mathbf{u}$ and of the wavevector $\mathbf{q}$, measured along the basic-cube axes, and $s=s(\mathbf{q}, \mathbf{u})$ is the corresponding velocity of sound.

If the crystal has cubic symmetry, and provided the 
$U_{0}^{ \pm}(\mathbf{r})$ are isotropic (in $\mathbf{r}$-space), the tensor $b_{i j}$ reduces to a scalar. The squared matrix element has the value :

$$
\left|\left\langle\mathbf{k}\left|V_{\text {def }}(\mathbf{q}, \mathbf{u})\right| \mathbf{k}+\mathbf{q}\right\rangle\right|^{2}=\frac{\hbar E_{\mathrm{c}}^{2}}{2 \rho s} q \cos ^{2}(\mathbf{q}, \mathbf{u})
$$

from which one finds that, for longitudinal polarizations, deformation-potential coupling dominates at high frequencies. It is, besides, zero for strictly transverse polarizations.

More complicated matrix elements obtain if the Bloch cell-functions $U_{\mathbf{k}}^{ \pm}(\mathbf{r})$ either depend upon $\mathbf{k}$ or no longer possess the spherical symmetry in r-space, an assumption underlying eq. (2.6). Such a situation occurs in InSb for conduction states of energy comparable to the gap, whose admixture with the valence states results in a partially P-like character of $U_{\mathbf{k}}^{ \pm}(\mathbf{r})$. The short-ranged deformation potential then admits of transitions induced by transverse phonons as well as of spin-flip transitions [10]. A numerical estimate [14] of these effects, however, indicates that they should indeed appear beyond the range of electron concentrations considered here (see $\S 6.1$ ).

2.2 EVAluation OF THE ABSORPTION RATES. The lifetime of an acoustic phonon interacting with a quasi-free election gas is easily calculated frora standard first-order perturbation theory [15]. If one assumes isotropy, but not necessarily parabolicity, of the conduction band, and defines the energydependent density-of-states effective mass $m$ by :

$$
\frac{1}{m(\varepsilon)}=\frac{1}{\hbar^{2} k} \times \frac{\mathrm{d} \varepsilon}{\mathrm{d} k}=\frac{2}{\hbar^{2}} \times \frac{\mathrm{d} \varepsilon}{\mathrm{d}\left(k^{2}\right)}
$$

the mean free path $\Lambda(\mathbf{q}, \mathbf{u})$ is given by the formula :

$$
\frac{1}{\Lambda(\mathbf{q}, \mathbf{u})}=\frac{m^{2}}{\pi \hbar^{4}} \times|V(\mathbf{q}, \mathbf{u})|^{2} \text { for } q<2 k_{\mathbf{F}}
$$

where $|V(\mathbf{q}, \mathbf{u})|^{2}$ is the squared matrix element of the phonon potential such as given by eq. (2.5) and (2.6). Let us recall that piezoelectricity and deformation potentials induced by a sinusoidal strain give additive contributions to $|V(\mathbf{q}, \mathbf{u})|^{2}$, since there is no interference term between them [16].

Eq. (2.8) is valid for all wavevectors $q$ inscribable in the Fermi sphere of energy $\varepsilon_{F}$ (condition of strong degeneracy). If not, a singularity reminiscent of the Kohn anomaly [17] is predictable when :

$$
q=2 k_{\mathrm{F}}\left[1+\frac{s}{v_{\mathrm{F}}}+3\left(\frac{s}{v_{\mathrm{F}}}\right)^{2} \times \frac{n}{m} \times \frac{\mathrm{d} m}{\mathrm{~d} n}\right] .
$$

In this equation, where $v_{\mathrm{F}}$ is the Fermi velocity, not only was the inelasticity included with the term $s / v_{\mathrm{F}}$, but also the dependence of the effective mass (at the Fermi level) upon the electron concentration $n$. However, $v_{\mathrm{F}}$ is usually much larger than the velocity of sound ( $s=0.03 v_{\mathrm{F}}$ at $n=10^{14} \mathrm{~cm}^{-3}$ ), and eq. (1.1) will be accurate enough for all cases of interest in this work.

A remarkable aspect of eq. (2.8) is the absence of explicit dependence upon the total number of electrons. Clearly, this is a consequence of Fermi-Dirac statistics at absolute zero. At finite temperatures, the Ziman formula taking account of emission processes [15] provides the correct solution, valid for any degeneracy. But, here again, this would cause negligible corrections at helium temperatures to the simplified result (2.8) because the Fermi level is so high in doped InSb $\left(\varepsilon_{\mathrm{F}}=500 \mathrm{~K}\right.$ for $\left.n=10^{17} \mathrm{~cm}^{-3}\right)$.

The simplest way to cope with the electron-electron interactions is to introduce a free-electron dielectric permeability [18] which will screen out the phonon potentials according to :

$$
\frac{V_{\text {screened }}(\mathbf{q}, \mathbf{u})}{V(\mathbf{q}, \mathbf{u})}=\frac{1}{1+\left(q_{\mathrm{TF}} / q\right)^{2}} F\left(\frac{q}{2 k_{\mathrm{F}}}\right)
$$

with

$$
F(x)=\frac{1}{2}+\frac{1-x^{2}}{4 x} \ln \left|\frac{1+x}{1-x}\right|
$$

The screening, or Thomas-Fermi, wavevector, $q_{\mathrm{TF}}$, is related to the Fermi wavevevector by :

$$
q_{\mathrm{TF}}^{2}=\frac{4 m e^{2} k_{\mathrm{F}}}{\pi \hbar^{2} \varepsilon_{\mathrm{r}}}
$$

while the Lindhard factor $F\left(q / 2 k_{\mathrm{F}}\right)$ is nearly equal to 1 for $q<2 k_{\mathrm{F}}$ and decreases abruptly through $\frac{1}{2}$ when $q=2 k_{\mathrm{F}}$. The screening-enhanced mean free path $\Lambda_{\mathrm{s}}(\mathbf{q}, \mathbf{u}, n)$ of a phonon interacting with an electron gas of density $n=k_{\mathrm{F}}^{3} / 3 \pi^{2}$ is then obtained by gathering eqs. $(2.5),(2.6),(2.10)$ into eq. $(2.8)$ to yield :

$$
\begin{aligned}
\frac{1}{\Lambda_{\mathbf{s}}(\mathbf{q}, \mathbf{u}, n)}= & \left(\frac{A}{q}+B q\right) \times \\
& \times \frac{1}{\left(1+C n^{1 / 3} q^{-2}\right)^{2} F^{2}\left(q / 2 k_{\mathbf{F}}\right)}
\end{aligned}
$$

with :

$$
\begin{aligned}
A & =\frac{32 \pi e^{2} m^{2}\left(e_{14}\right)^{2}}{\hbar^{3} \varepsilon_{\mathrm{r}}^{2} \rho s} \times \\
B & \times\left(\bar{q}_{x} \bar{q}_{y} \bar{u}_{z}+\bar{q}_{y} \bar{q}_{z} \bar{u}_{x}+\bar{q}_{z} \bar{q}_{x} \bar{u}_{y}\right)^{2}, \\
& =\frac{m^{2} E_{\mathrm{c}}^{2}}{2 \pi \hbar^{3} \rho s} \cos ^{2}(\mathbf{q}, \mathbf{u}) \\
C & =\left(\frac{3}{\pi}\right)^{1 / 3} \times \frac{4 e^{2} m^{2}}{\hbar^{2} \varepsilon_{\mathrm{r}}} .
\end{aligned}
$$

We have evaluated numerically $\Lambda_{\mathrm{s}}$ with, for the physical parameters entering eq. (2.12), the following values : $e_{14}=2.13 \times 10^{4} \mathrm{dyne}^{1 / 2} \times \mathrm{cm}^{-1}$ for the piezoelectric modulus [19], $E_{\mathrm{c}}=13 \mathrm{eV}$ for the deformation potential constant $[16,20], \varepsilon_{\mathrm{r}}=17$ for 
the lattice dielectric permeability, $\rho=5.80 \mathrm{~g} / \mathrm{cm}^{3}$ at $T=0$. For computational convenience, we used, for the density-of-states effective mass, an empirical interpolating formula :

$$
\frac{m}{m_{0}}=1.35 \times 10^{-2}+1.66 \times 10^{-11}\left[n\left(\mathrm{~cm}^{-3}\right)\right]^{1 / 2}
$$

which fits the experimental values of the literature [20, 21 ] to within about $2 \%$ throughout the concentration range $10^{13}$ to $5 \times 10^{19} \mathrm{~cm}^{-3}$.

To be specific, we considered the case of the purely longitudinal phonon propagating along [111] and causing electron transitions by its screened piezoelectric and deformation potential fields. The choice of the corresponding velocity of sound, $s=3.92 \mathrm{~km} / \mathrm{s}$, and of the angular factors of $A$ and $B$ in eq. (2.12) results from the work of section 4 , table II. The mean free paths, as a function of $n$, are plotted in figure $1 a$ (full curves) for several values of the phonon energy $(2,4,10$ and $20 \mathrm{~K})$. The dashed curves correspond to the same calculations performed without the screening effects included. Let us focus our attention for instance on the full curve corresponding to an energy of $4 \mathrm{~K}$ : the free path is infinite till a concentration is reached where $2 k_{\mathrm{F}}=q$. Then, at intermediate concentrations, it stays on a plateau according to (2.8), slightly decreasing due to the variation of the effective mass, and eventually increases again as the screening effects take over. For large enough energies, however, screening is less efficient and the $\Lambda_{\mathrm{s}}(n)$ curve never rises. Let us also note that the correcting factor $F\left(q / 2 k_{\mathrm{F}}\right)$ introduces no more than a hardly visible rounding of the (full) curves around the value $2 k_{\mathrm{F}}=q$.

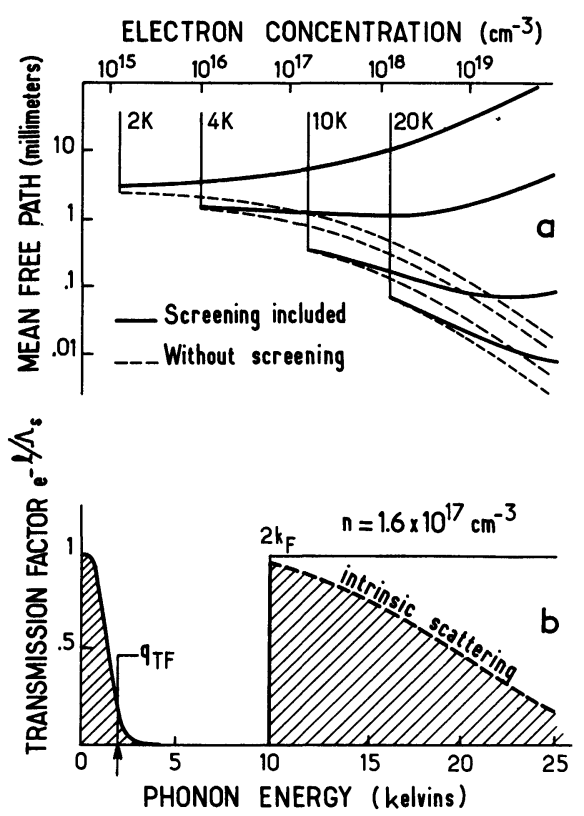

FIG. 1. - (a) Calculated mean free paths for longitudinal phonons of energies : 2, 4, 10 and $20 \mathrm{~K}$ as a function of the electron concentration. (b) Transmission factor of $L$ phonons versus energy for a doped sample of length $9 \mathrm{~mm}$, with Rayleigh scattering included.
An alternative way of viewing these results is shown in figure $1 b$, in which the transmission factor of a semiconducting slab is plotted as a function of the phonon energy for a given value of the electron concentration $\left(n=1.6 \times 10^{17} \mathrm{~cm}^{-3}\right.$; cut-off energy : $10 \mathrm{~K})$. We have plotted the function $\exp -\left(l / \Lambda_{\mathrm{s}}\right)$ taking for the propagation length $l=0.9 \mathrm{~cm}$. It illustrates the filtering function of a doped semiconductor: the electron gas is transparent to the phonons such that either $q>2 k_{\mathrm{F}}$, or, due to the screening effects, $q \ll q_{\mathrm{TF}}$. For the transmission of the high frequency phonons, one should also take into account the intrinsic (independent of the doping) scattering, which, at low temperatures, is mainly due to the isotopic distribution and contributes to the reciprocal mean free path a term proportional to $q^{4}$. According to thermal conductivity measurements [22], the mean free path associated with this Rayleigh scattering equals $1 \mathrm{~cm}$ when $q=7.5 \times 10^{6} \mathrm{~cm}^{-1}$, this value was inserted to calculate the dashed curve in figure $1 b$.

3. The heat pulse method. -3.1 GenERAL DESCRIPTION. - The heat-pulse configuration derives from reference [1]. A piece of a single crystal, immersed in liquid helium, is provided on one end with a metal film whose mechanical vibrations (phonons) are excited by short electrical pulses (typically $1 \mathrm{~W}$ during $10^{-7} \mathrm{~s}$ ). These, once transmitted to the crystal, divide into several wave-fronts, one for each polarization, and travel with little diffusion. They are detected at the other end by a temperature-dependent resistor. For optimum response, the phonon emitter and detector are thin metal films fabricated by vacuum evaporation.

The task of determining the generated frequency spectrum is a difficult one, either theoretically or experimentally. One has to analyze the creation, possibly dependent upon the film thickness, of the phonons generated by the electron current, and their transmission at the solid-solid interface, the physical structure of which is not well known on a microscopic scale (let us recall that a typical phonon wavelength is $100 \AA$ ). A convenient assumption [1] is that the emitter film is heated up to a certain temperature $T_{\text {em }}$ above the substrate temperature $T_{0}$ and radiates phonon with a power spectrum $P(q)$ :

$$
P(q) \propto q^{3}\left[N_{q}\left(T_{\mathrm{em}}\right)-N_{q}\left(T_{0}\right)\right]
$$

with

$$
N_{q}(T)=\left[\exp \left(\frac{\hbar q s}{k_{\mathrm{B}} T}\right)-1\right]^{-1}
$$

which has the Planck form. Power is radiated at the total rate :

$$
\dot{Q}=\eta \sigma\left(T_{\mathrm{em}}^{4}-T_{0}^{4}\right)
$$


where $\sigma$ is the Stefan constant appropriate to phonon emission, viz.

$$
\sigma=\frac{\pi^{2} k_{\mathrm{B}}}{120 \hbar^{3}}\left(\frac{1}{s_{\mathrm{L}}^{2}}+\frac{1}{s_{\mathrm{FT}}^{2}}+\frac{1}{s_{\mathrm{ST}}^{2}}\right)
$$

and $\eta(0<\eta<1)$ a mean coefficient which measures the acoustic mismatch between the metal film and the substrate [23]. $s_{\mathrm{L}}, s_{\mathrm{FT}}, s_{\mathrm{ST}}$ represent the velocities of sound in the emitter film corresponding respectively to the longitudinal, fast transverse and slow transverse polarizations. This model is referred to as the blackbody radiation model, corrected for the acoustic mismatch.

More recent calculations [24] take into account the finite value of the phonon decay time $\tau_{\mathrm{e}-\mathrm{ph}}(q)$, limited by the electrons in the emitter, with respect to the escape time $\tau_{\mathrm{h}}$ from the film. Unlike the electrons, which are heated up to a (power-dependent) temperature $T_{\mathrm{e}}$, the phonon distribution can not be characterized by a single temperature. As compared to the blackbody model, for which $\tau_{\mathrm{e}-\mathrm{ph}} \ll \tau_{\mathrm{b}}$, whatever $q$, the spectrum here obtained looks closer to that primarily generated by the Joule effect. The main difficulty associated with this model is obviously to assign definite values to the parameters $\tau_{\mathrm{b}}$ and $\tau_{\mathbf{e}-\mathbf{p h}}$.

Previous experimental tests of the theory have been of two kinds. Firstly, if one admits the validity of some radiation model (blackbody for instance), the parameter $T_{\mathrm{em}}$, as determined by eq. (3.2), can be checked against a direct measurement of the film temperature. This measurement was done [23, 25] by monitoring the transient resistance of the film during the application of the pulse. The results for several cases of solid-solid interfaces support the blackbody model corrected for the acoustic mismatch. However, in the range of moderate input powers $\left(T_{\mathrm{em}}<10 \mathrm{~K}\right)$, the transient measurements of resistance deviations are not reliable and no conclusion can be drawn.

A more sensitive method consists in probing the spectrum emitted into the substrate with resonant scatterers. Thus, the heat-pulse attenuation by the vibronic levels $(250 \mathrm{GHz})$ of $\mathrm{V}^{+++}$in $\mathrm{Al}_{2} \mathrm{O}_{3}$ and its power dependence were found to be consistent with the blackbody model [6]. Tunability was achieved thanks to the paramagnetic $\mathrm{Fe}^{++}$ions in $\mathrm{MgO}$; there, the importance of the interfacial acoustic mismatch in determining $T_{\mathrm{em}}$ was recognized [7]. However, due to the limited accuracy of heat pulse measurements, heavy concentrations of scatterers were taken to obtain sensible attenuation (up to $50 \%$ ), thus resulting in a large effective bandwidth of the transmission medium. Besides, by finely monitoring the dichroism of $\mathrm{Tm}^{++}$ions in $\mathrm{SrF}_{2}$, other workers could preserve the narrow bandwidth of the spinphonon system [8], and present the phonon densities as a function of the frequency. The data agree well with the blackbody assumption, although direct comparison with others is impossible since the generator was not in intimate contact with the substrate, but rather bonded by glue.

For most experiments, we used bolometric phonon detectors made of superconducting films ( $\mathrm{Sn}, \mathrm{Al}$ ) working at their transition temperature [1]. When a frequency discriminator was required (see section 5), the heat pulse signals were detected by superconducting tunnel junctions $[2,26]$.

3.2 EXPERIMENTAL TECHNIQUeS. - The InSb samples were tellurium doped single crystals. They were X-ray oriented along a symmetry direction $( \pm 1$ degree), and cut with a spark erosion machine. Then, they were laid on a fine cloth bathed in an alumina suspension (down to $200 \AA$ ) and set into gentle motion on a special vibrating machine (Wirtz). It is clear that the mode of preparation, either sandblasting, polishing, etching or cleaving, has consequences on the phonon properties at the surface. However, the quality of the polish attainable with our means was not found to alter significantly the heat pulse amplitude measurements.

Insulation of both ends of the crystals was made by vacuum deposition around $10^{-6}$ torr of a Pyrex layer, $8000 \AA$ thick on the emitter side, $4000 \AA$ thick on the detector side. Care was taken to fabricate the thin films in the same conditions of evaporation rates and thicknesses for all the samples to permit comparisons. The $\mathrm{SiO}$ powder was thoroughly degased in its tungsten crucible during about $15 \mathrm{~min}$., each time, before deposition. The emitter (gold : $400 \AA$ ) and the detector (most often, tin : $2000 \AA$ ), were evaporated along the shape of a coil (overall area : $1.5 \times 1.5 \mathrm{~mm}^{2}$ ). They were then connected to the electrical circuits by short silver leads (diameter : $0.1 \mathrm{~mm}$ ) stuck with a suspension of silver powder. The transducers (emitter and detector) has their size minimized because they form through the SiO layer a non-negligible capacitive shunt to the earthed sample. This precaution proved very important in accurate time-of-flight measurements [5, 38]. Finally, the two sides of the sample were separated by an aluminum foil to screen out the initial electromagnetic feedthrough.

The instrumental set-up is the standard one. A fast generator delivers electrical pulses of up to $1 \mathrm{~A}$ to the emitter gold film. For quantitative comparisons between two different samples, the duration and power of the input pulses have to be carefully adjusted by appropriate pulse techniques. This is necessary for two reasons : the random values of the emitter impedances $( \pm 10 \%$ around $50 \Omega)$, and the fact that one is usually considering response signals which have not reached the steady state. The bolometer signals are sent to a wide-band amplifier (Keithley : Type 107) through a $50 \Omega$ coaxial system, and then fed into a boxcar integrator (P.A.R. 160) whose output is plotted as a function of the time. For the same reason as before, the boxcar aperture was kept 
constant ( $25 \mathrm{~ns})$. All data were finally extracted from the complete paper recordings of the bolometer signals. Possible shifts of the baseline or large fluctuations of various origins are thus readily recognized. Provided these natural prescriptions are observed, the reproducibility of the relative (longitudinal over transverse) amplitude measurements, on a given sample, is better than usually quoted, and ranges within $5 \%$ for one experiment to the other.

The use of tin bolometers at their superconducting transition was possible from $3.7 \mathrm{~K}$ down to $1.25 \mathrm{~K}$ thanks to a mechanical pumping system (Alcatel : $220 \mathrm{~m}^{3} /$ hour), with a magnetic field to tune the transition temperature. The field, applied parallel to the films, was produced by superconducting coils and used for the regulation. Field control rather than temperature control proved easier in operation. In the transition, the temperature coefficient of the bolometer resistance $\left(R^{-1} \mathrm{~d} R / \mathrm{d} T \simeq 5\right)$, of about $20 \mathrm{mV}$ per kelvin, is not a perfectly reproducible parameter. This is a serious limitation of the heat pulse method in general, but a procedure such as that described in paragraph 5.1 can overcome this difficulty.

4. Consideration of acoustic anisotropy. - The dispersion relations of low frequency acoustic waves in a crystal of cubic symmetry (InSb) are entirely specified by the set of the three second-order elastic constant $C_{11}, C_{12}, C_{44}$, and the mass density $\rho$ $\left(\rho=5.80 \mathrm{~g} / \mathrm{cm}^{3}\right.$ in $\mathrm{InSb}$ at $\left.0 \mathrm{~K}\right)$. For a given wavevector $\mathbf{q}$, of director cosines $\bar{q}_{x}, \bar{q}_{y}, \bar{q}_{z}$, the polarizations of the normal modes are the eigenvectors of the matrix $[27,28]$ :

$$
\left\|\begin{array}{lll}
K \bar{q}_{x}^{2} & \bar{q}_{x} \bar{q}_{y} & \bar{q}_{x} \bar{q}_{z} \\
\bar{q}_{y} \bar{q}_{x} & K \bar{q}_{y}^{2} & \bar{q}_{y} \bar{q}_{z} \\
\bar{q}_{z} \bar{q}_{x} & \bar{q}_{z} \bar{q}_{y} & K \bar{q}_{z}^{2}
\end{array}\right\|
$$

where $K=\left(C_{11}-C_{44}\right) /\left(C_{12}+C_{44}\right)$. The phase velocities $(s)$ are related to the corresponding eigenvalues $(r)$ by :

$$
\rho s^{2}=C_{44}+\left(C_{12}+C_{44}\right) r .
$$

The normal modes propagating along the symmetry directions are either purely longitudinal or purely transverse, with degeneracy of the transverse modes along [100] and [111]. For $\mathbf{q}$ along [110], the two transverse modes, referred to as FT (fast transverse) and ST (slow transverse), are polarized respectively along [001] and [11̄0].

The heat pulse method enables one to visualize the acoustic anisotropy straightforwardly. A pure InSb crystal was cut to almost equal lengths $(1.85 \pm 0.05 \mathrm{~cm})$ along the symmetry directions and heat pulses (Fig. 2) were detected by superconducting bolometers. We shall now use these plots to determine the elastic constants.

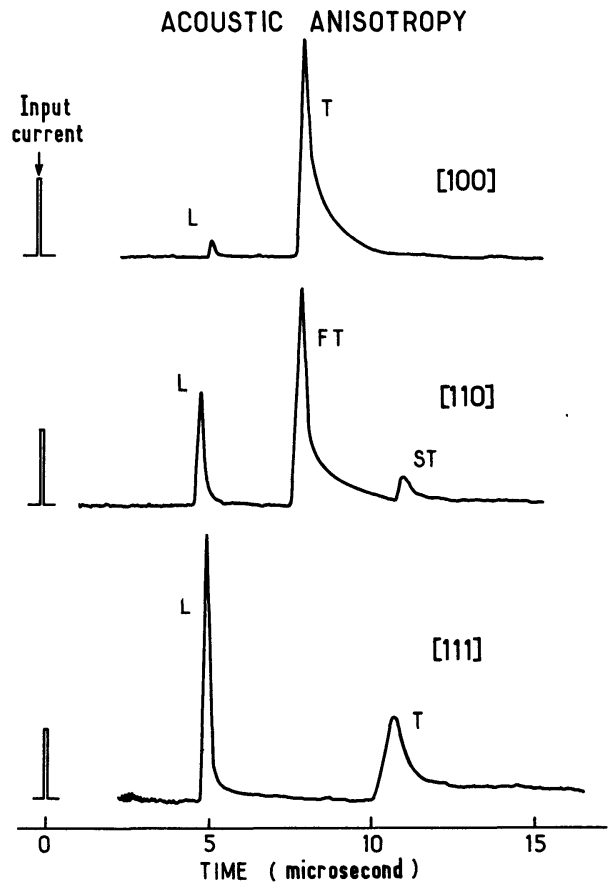

Fig. 2. - Heat pulse signals detected after approximately equal propagation lengths $(1.85 \pm 0.05 \mathrm{~cm})$ along the principal directions of a pure InSb single crystal. Horizontal time scale corresponds to the [111] experiment (length : $1.875 \mathrm{~cm}$ ).

The relationship of $\rho s^{2}$ to the elastic constants according to eq. (4.2) are recalled for the principal modes in the second column of table I. In the fourth

\section{TABLE I}

Velocities of sound (s) computed from eq. (4.3), and

\begin{tabular}{|c|c|c|c|c|}
\hline \multicolumn{2}{|c|}{ Mode } & $\rho s^{2}$ (theory) & $s$ & $V_{\text {heat }}$ \\
\hline & & - & - & - \\
\hline [100] & $\begin{array}{l}\mathrm{L} \\
\mathrm{T}\end{array}$ & $\begin{array}{l}C_{11} \\
C_{44}\end{array}$ & $\begin{array}{l}3.475 \\
2.335\end{array}$ & $\begin{array}{l}3.49 \\
2.345\end{array}$ \\
\hline [110] & $\begin{array}{l}\text { L } \\
\text { FT } \\
\text { ST }\end{array}$ & $\begin{array}{l}\left(C_{11}+C_{12}+2 C_{44}\right) / 2 \\
C_{44} \\
\left(C_{11}-C_{12}\right) / 2\end{array}$ & $\begin{array}{l}3.835 \\
2.335 \\
1.680\end{array}$ & $\begin{array}{l}3.85 \\
2.345 \\
1.67\end{array}$ \\
\hline [111] & $\begin{array}{l}\mathrm{L} \\
\mathrm{T}\end{array}$ & $\begin{array}{l}\left(C_{11}+2 C_{12}+4 C_{44}\right) / 3 \\
\left(C_{11}-C_{12}+C_{44}\right) / 3\end{array}$ & $\begin{array}{l}3.945 \\
1.920\end{array}$ & $\begin{array}{l}3.92 \\
1.87\end{array}$ \\
\hline
\end{tabular}
measured heat-pulse velocities $\left(V_{\text {heat }}\right)$, in $\mathrm{km} / \mathrm{s}$

column, the measurements of heat-pulse velocities are reported. Then, a set of elastic constants was sought in order that the computed values of $s$ (third column) fit the measured values (for reasons to be developed soon, along [111], we put the transverse polarizations from this comparison). The best agreement was obtained with the following choice (in units of $10^{12}$ dynes $/ \mathrm{cm}^{1 / 2}$ ) :

$$
\begin{aligned}
& C_{11}=0.700 \\
& C_{12}=0.373 \\
& C_{44}=0.316 .
\end{aligned}
$$


Potter's ultrasonic measurements [29], extrapolated to zero $K$, gave respectively : $(0.666),(0.335)$ and (0.314). Those more recently of Slutsky and Garland [30] gave : (0.691 8), (0.378 8) and (0.313 2), in much closer agreement with the values reported here.

The transit times of the heat pulses could be measured with an accuracy of $0.5 \%$, while the uncertainty of sample length and of time-base linearity (Boxcar time-base was calibrated against a synthetized $2 \mathrm{MHz}$ sine-wave) were of the same order. Since our measurements were repeated many times, we think that results (4.3) can be credited better than $1 \%$ accuracy. On the other hand, internal consistency of the heatpulse results can be checked by noting that 6 independent velocity measurements (the $T$ polarization along [111] still being discarded) can be accounted for within $0.6 \%$ in terms of only three parameters.

Since the bolometer is a quadratic detector, the line joining it to the emitter defines a ray or energy direction rather than a q-direction. Energy propagation is governed by the group-velocity :

$$
\mathbf{v}(\mathbf{q})=\frac{\mathrm{d} \omega}{\mathrm{dq}}
$$

which differs from the phase velocity $\mathbf{s}=\omega \mathbf{q} / q^{2}$ except in symmetry directions. The acoustic anisotropy of a crystal is best described by the wave-surface [28], locus of $\mathbf{s}(\mathbf{q})$, and its inverse, the slowness surface. Both are separable into three sheets, one per polarization. Differenciation of $\mathbf{q} / \omega(\mathbf{q})$ with respect to $\mathbf{q}$, plus scalar multiplication by $\mathbf{v}$ yields a factor $\omega-\mathbf{q} \cdot(\mathrm{d} \omega / \mathrm{dq})$ which is zero whenever the proportionality relation between $\omega$ and $|\mathbf{q}|$ holds, that is for long waves. As a result, $\mathbf{v}$ is normal to the slowness surface and its amplitude satisfies :

$$
s(\mathbf{q})=|\mathbf{v}(\mathbf{q})| \cos (\mathbf{q}, \mathbf{v}) .
$$

In a cubic crystal, the group velocities are also related to the eigenvalues of (4.1) by the useful Musgrave formula [28] :

$$
\rho v_{\alpha}(\mathbf{q}) s(\mathbf{q})=C_{44} \bar{q}_{\alpha}+\left(C_{12}+C_{44}\right) r \frac{\bar{u}_{\alpha}^{2}}{\bar{q}_{\alpha}}
$$

where $\alpha$ indexes the cartesian components of $\mathbf{v}, \mathbf{q} /|\mathbf{q}|$, and $\mathbf{u}$ along the cube axes.

The section of the wave surface (full curves) and of the slowness surface (dotted curves) by a (110) plane are drawn in figure 3. Numerical computation with the values (4.3), and plotting, were performed by a 9810 A Hewlett-Packard programmable calculator. The analytical problem is rendered easy because, in the eigenvalue equation, the term corresponding to the [1 10 ] polarization $T_{2}$ factorizes out (its slowness curve is an ellipse).

The q-vectors giving energy propagation along the [111] direction, which was given the greatest attention in this work, were found by searching in

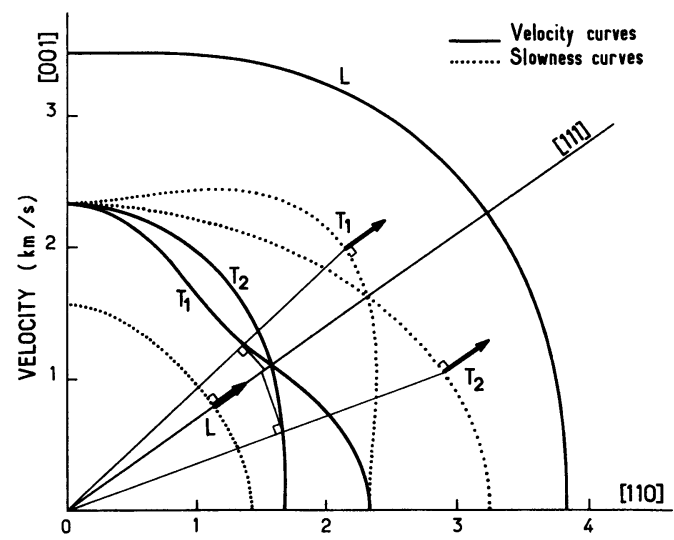

Fig. 3. - Computer plot of the intersections of the velocity and

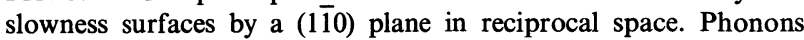
contributing to the $T$ heat pulse along [111] arise from the double arrows marked $T_{1}$, polarized in the (110) plane, and $T_{2}$, polarized along [110].

successive approximations the points of the slowness surface whose normals point to that direction. These are indicated by the double arrows noted $L$, and $T_{1}$ and $T_{2}$ (off-axis), in figure 3 . This exhausts all the possibilities since the points out of the (110) plane are located in the equivalent planes (10) $)$ and $(01 \overline{1})$ [31]. It is seen that the group-velocities of $T_{1}$ and $T_{2}$, resulting from eq. (4.6) are not equal to $s$ along [111]. If we come back to table I, we note that the measured velocity of the $T$ pulse along [111], $1.87 \mathrm{~km} / \mathrm{s}$, is very close to the largest group-velocity $(1.878 \mathrm{~km} / \mathrm{s}$ calculated for the $\mathrm{T}$ mode). Unfortunately, it was not possible experimentally to separate the arrival of the two transverse modes.

Figure 3 also teaches us, at least qualitatively, how much amplitude must be expected for each polarization. For instance, the ST heat pulse propagating along [110] originates from the sharp tip of the slowness ellipse, while the FT pulse comes from qvectors as far as 23.6 degrees from the [110] axis. This enhancement of anisotropy, or self-focusing effect, is responsible for the very large amplitude ratio FT/ST observed in figure 2 . The striking difference between the heat-pulse signals propagating along [100] and [111] can be explained on the same basis.

Our aim is finally to compute the interaction matrix elements, for which purpose we need to know the exact polarizations associated with each mode. For q along [111], we first remark that the group velocity of the $L$ mode is parallel to $\mathbf{q}$ : in effect, the transverse modes are degenerate and this is possible only if their eigenspace is orthogonal to [111] due to the thirdorder symmetry of the cube. The $T$ modes are thus purely transverse and consequently also the $L$ mode. The equality (and parallelism) of $v$ (longitudinal) to the phase velocity then follows. On the contrary, $T_{1}$ and $T_{2}$ are not pure transverse. The relevant parameters, as well as the angular factors of the squared matrix elements (2.5) and (2.6), are listed in table II. 
TABLE II

Angular factors of the matrix elements for phonon absorption. $v$ is the group-velocity in the [111] direction. $\Sigma$ is for cyclic permutation

$\begin{array}{cccccccc}\text { Mode } & \theta \text { (degrees) } & v(\mathrm{~km} / \mathrm{s}) & \bar{u}_{x} & \bar{u}_{y} & \bar{u}_{z} & \cos ^{2}(\mathbf{q}, \mathbf{u}) & \left(\Sigma \bar{q}_{x} \bar{q}_{y} \bar{u}_{z}\right)^{2} \\ - & - & - & - & - & - & - & - \\ L & 35.26 & 3.945 & 1 / \sqrt{3} & 1 / \sqrt{3} & 1 / \sqrt{3} & 1 & 1 / 3 \\ T_{1} & 42.87 & 1.878 & 0.459 & 0.459 & -0.761 & 0.0018 & 0.014 \\ T_{2} & 20.10 & 1.833 & 1 / \sqrt{2} & -1 / \sqrt{2} & 0 & 0 & 0\end{array}$

We notice that the $T_{1}$ mode polarized in the (1110) plane has a slight longitudinal component which, however, makes a negligible contribution to the deformation potential interaction. The piezoelectric attenuation can be ignored as well. Since the $T_{2}$ mode is not coupled at all, this confirms the previous assertion that $T$ waves do not interact with the electrons, either by deformation potential or by piezoelectric coupling. For the $L$ mode, one has to take both couplings into account as it was done in section 2 (Fig. 1).

5. Illustration of the theory. - Through simpleminded heat-pulse experiments, some salient features of the theory are accessible, for one disposes of generators of high-frequency phonons (the Joule emitters), of a polarizer (time-of-flight separation of the modes) and of frequency discriminators (superconducting tunnel junctions).

5.1 Selective COUPLING OF THE LONGITUdiNAL MODES [3], [32]. - The statement that transverse phonons are not attenuated :

$$
\alpha_{\mathrm{T}}(q) \equiv 0
$$

by the electrons in a semiconductor results from the deformation potential assumption. It is far from general, however, in a real crystal, even of the cubic symmetry, owing to the complexity of the band structure, but it is true at high phonon frequencies in n-type III-V materials (see section 2). If the phonon frequency is too low, the deformation potential coupling may be overpowered by the piezoelectric effects and eq. (5.1) will not hold true. This was the case for the attenuation of $9 \mathrm{GHz}$ hypersonic waves in InSb [33] and also for the acousto-electric amplification of up to $150 \mathrm{GHz}$ phonons in $\mathrm{GaAs}$ [4], where piezoelectricity is particularly strong. In InSb, the deformation potential interaction dominates [16] above $20 \mathrm{GHz}$ (or $1 \mathrm{~K}$ ) so that $(5.1)$ can be tested with thermal phonons. For the direction of propagation we chose, with energy velocity along [111], the mean free path of $T$ waves limited by the electrons should, according to table II, be of at least $10 \mathrm{~cm}$ whatever the frequency. Therefore, the attenuation of $T$ waves by electrons is negligible on our scale.

The heat-pulse signals displayed in figure 4 correspond to a set of samples of widely different concen- trations. Increasing the doping causes a strong reduction of the $L$ pulses (compared to the $T$ pulses). The true nature of this effect may be worth reminding. It is not the result of an increased attenuation coefficient $\alpha_{\mathrm{L}}$ since, to a large extent, this quantity is independent of $n$ as previously noted. Rather, as $2 k_{\mathrm{F}}$ proceeds across the phonon spectrum (see inset of Fig. 4), a smaller and smaller part of the broadband phonon source is selected.

\section{In Sb [111]}
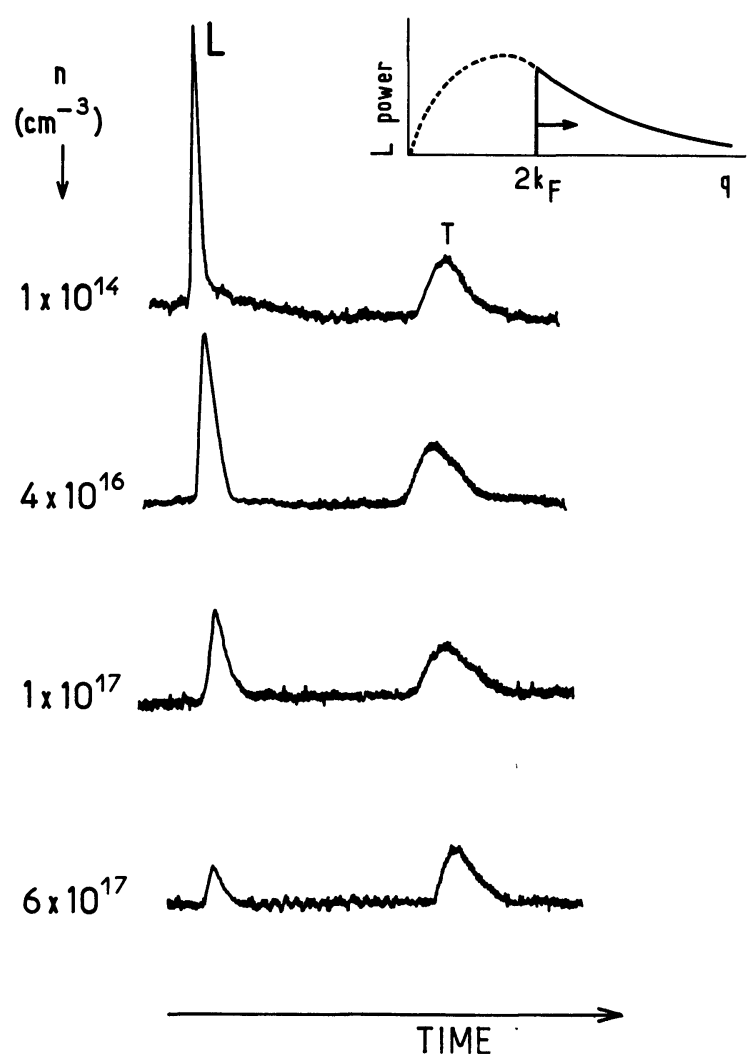

FIG. 4. - Boxcar tracings of $L$ and $T$ heat pulses detected by a tin bolometer, with $T$ amplitudes approximately normalized. Input power : $60 \mathrm{~W} / \mathrm{cm}^{2}$; duration : $10^{-7} \mathrm{~s}$. Inset explains the gradual $L$ attenuation as $2 k_{\mathrm{F}}$ proceeds into the phonon spectrum.

This experiment shows clearly that $\alpha_{\mathrm{L}} \gg \alpha_{\mathrm{T}}$. But it is not suited to verify eq. (5.1) in an absolute manner, because the thin-film detectors do not have reproducible sensitivities [3], even when fabricated 
in identical conditions. Calibrating the bolometers was therefore necessary for this and future applications. Pulses of second sound in the surrounding helium bath $(1.25 \mathrm{~K})$ served this purpose.

According to the arrangement of figure 5, two samples are examined at the same time. Their bolo-

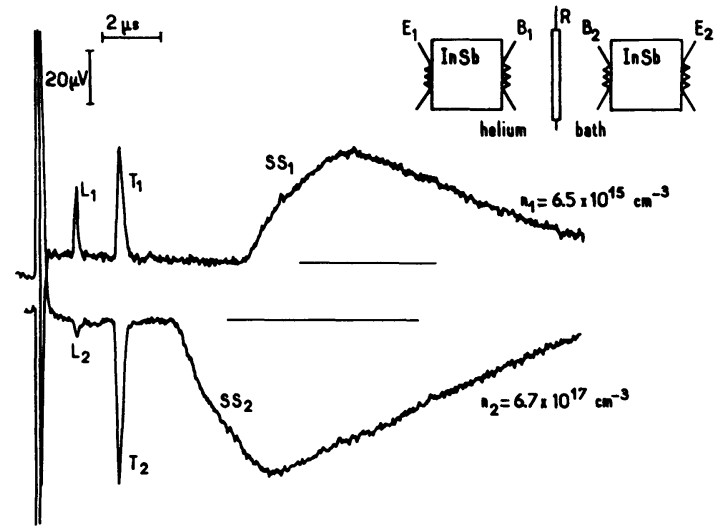

FIG. 5. - Inset : symmetric arrangement of two InSb samples of concentrations $n_{1}$ and $n_{2}$. Equal second sound pulses emitted into the helium bath by the source $\mathrm{R}$ give on the bolometers $\mathrm{B}_{1}$ and $B_{2}$ the responses $S_{1}$ and $S_{2}$ (the time shift between them has no direct meaning). $L_{1}, T_{1}$ and $L_{2}, T_{2}$ are the responses to equal heat pulses emitted by $E_{1}$ and $E_{2}$ respectively, and travelling in the InSb samples.

meters $B_{1}$ and $B_{2}$ are placed in front and at an equal distance $(9.5 \pm 0.1 \mathrm{~mm})$ from a thin-film rod resistor, source of a second-sound (SS) pulse. This signal is detected by the superconducting bolometers $\left(\mathrm{SS}_{1}\right.$ and $\mathrm{SS}_{2}$ ) as well as the usual heat pulses having travelled in the InSb crystals. The responses $\mathrm{SS}_{1}$ and $\mathrm{SS}_{2}$, which, by symmetry, correspond to the same temperature excursion, give a measure of the sensitivity of each detector. This calibration method (for details, see [32]) seems to the authors more accurate than a measurement of the temperature coefficient of the bolometers. Now, with reference to the SS pulses, we note that the $T$ pulses for two $\mathrm{InSb}$ samples of quite different dopings are equal, which indicates a strict observance of the selection rule $\alpha_{\mathrm{T}} \equiv 0$. While strongly suggested by theory, this result is not trivial in two respects :

(i) We do not know of any previous clearcut experimental proof. As we mentioned previously, it has not been possible to reach the deformation potential range with coherently generated phonons. On the other hand, the fitting of $\alpha_{L}$ and $\alpha_{T}$ to thermal conductivity data [34] for instance is a rather indirect way to attack this problem.

(ii) It shows that impurities in concentration of up to $6.7 \times 10^{17} \mathrm{~cm}^{-3}$ produce negligible phonon attenuation as compared to intrinsic (isotope) scatterers. The same conclusion was reached thanks to a detailed study of the heat conductivity in InSb as a function of the doping and of the compensation [35]. This consideration will be of primary importance in further measurements to follow, where the $T$ pulses will serve as calibrators of the heat-pulse signals.

5.2 FERMI-SURFACE CUT-OFF. - In order to examine the frequency dependence of the attenuation coefficient $\alpha_{\mathrm{L}}$, we used superconducting tunnel junctions. These thin-film devices [26] detect only the phonons of energy larger than the superconducting gap $2 \Delta(2 \Delta=1.145 \mathrm{meV}$ for tin at $1.25 \mathrm{~K})$. Consider the $L$ energy spectrum (Fig. 6 , inset) emitted by the gold film at a given input power. Only the shaded area
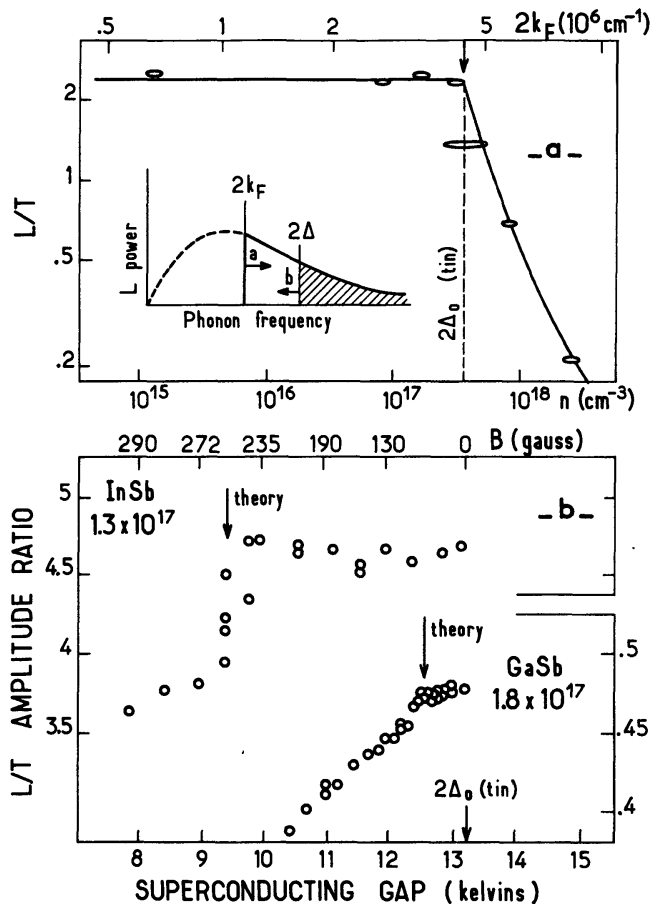

Fig. 6. - Inset : in the transmitted spectrum, all phonons above $2 k_{\mathrm{F}}$ are present, but only those of energy $\geqslant 2 \Delta$ are detected by the tin tunnel junction. (a) Ratio of the $L$ and $T$ signals as a function of the electron concentration $n$; power : $4 \mathrm{~W} / \mathrm{cm}^{2}$. (b) $L / T$ for two n-type crystals of InSb and $\mathrm{GaSb}$ as a function of the superconducting gap tuned by a magnetic field.

above $2 \Delta$ is detected by the tin diode, while filtering by the electrons limit the low-frequency side at $2 k_{\mathrm{F}}$, according to eq. (1.1). Thus, a Kohn-anomaly of the detected signal is expected to appear when :

$$
\hbar s \times 2 k_{\mathrm{F}}=2 \Delta \text {. }
$$

There are several ways of fulfilling this condition :

(i) If the electron concentration is increased from low values, the detectable phonons ( $\hbar q s>2 \Delta$ ) are untouched, i.e., the $L$ detected signal remains constant, until $n$ reaches the critical value (marked by a dotted line in figure $6 a$ ) such that eq. (5.2) is satisfied. Then, the signal goes decreasing as the doped samples transmit fewer and fewer detectable phonons. The singularity one observes is quite of the same origin as the Kohn effect reported in the phonon dispersion relations of metals [17, 36], but here 
concerns phonon lifetimes rather than phonon energies.

(ii) The energy gap of the detecting junctions can be tuned by application of a magnetic field. We operated on samples having an $L$ phonon energy at $2 k_{\mathrm{F}}$ smaller than the gap $2 \Delta_{0}$ of tin in zero field. In a field $(H)$ the gap $2 \Delta(H)$ is swept to lower values. Therefore, the detected signal should at first be regular, then present a singularity and be regular again. This is indeed the behaviour exhibited in figure $6 b$ by the two samples of $\operatorname{InSb}\left(n=1.3 \times 10^{17} \mathrm{~cm}^{-3}\right)$ and $\mathrm{GaSb}$

$$
\left(n=1.8 \times 10^{17} \mathrm{~cm}^{-3}\right) \text {. }
$$

The location of the singularity is moreover in agreement with the theoretical values of $n$ being determined by Hall effect measurements (for a fuller description of this experiment, see ref. [5]).

Let us remark that in heat-conductivity measurements, where the mean phonon frequency is tuned by varying the temperature, the Fermi-surface cut-off does not usually show [35], except, to a certain extent, in heavily doped p-type samples [37]. Here, the cut-off appears distinctly because a frequency discriminator is available in the thermal range.

5.3 SCREENING EFFECTS. - The consequences of static screening were calculated in section 2. From eq. (2.10), they are prominent on long waves. As a result (see inset of Fig. 7, which is an adaptation of Fig. $1 b$ ), two phonon windows appear in the absorption spectrum $\left(q \ll q_{\mathrm{TF}}\right.$, the screening parameter, and $q>2 k_{\mathrm{F}}$ ). For sufficiently high concentrations, these two groups of phonons will have, on account of chromatic dispersion, distinct velocities of propagation, whose resolution is within the capabilities of heat pulse-techniques [38].

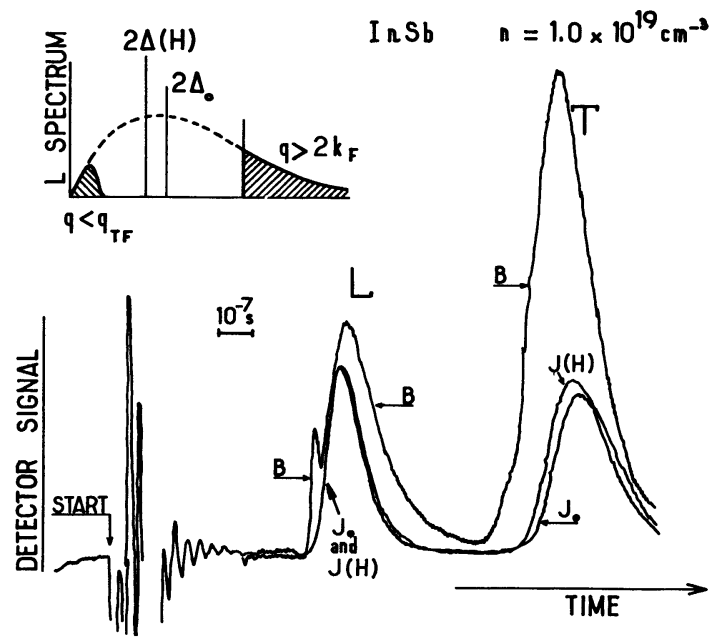

FIG. 7. - Heat pulse signals along [111] for a heavily doped sample (length : $2.14 \mathrm{~mm}$ ) detected by a tin bolometer (B trace), by a tin tunnel junction, $J_{0}$, and the same junction in a magnetic field, $J(H)$. Note the dispersion effects and the structure appearing on the (B) longitudinal signal due to the screened low-frequency phonons ( $\left.q<q_{\mathrm{TF}}\right)$. Inset shows the two groups of transmitted phonons $q<q_{\mathrm{TF}}$ and $q>2 k_{\mathrm{F}}$
Figure 7 shows the signals detected after propagation in a heavily doped sample $\left(n=1.0 \times 10^{19} \mathrm{~cm}^{-3}\right)$ for which $q_{\mathrm{TF}}=2.6 \times 10^{6} \mathrm{~cm}^{-1}$ and

$$
2 k_{\mathrm{F}}=1.33 \times 10^{7} \mathrm{~cm}^{-1} \text {. }
$$

Three traces are present : $B$ was measured by a tin bolometer, $J_{0}$ with an $\mathrm{Sn} / \mathrm{Sn}$ oxide/Sn tunnel junction and $J(H)$ was taken with the same diode tuned by a small magnetic field. The dispersion effects are quite apparent on the $T$ signals : the junction signal $J_{0}$ is delayed compared to the $B$ signal because the group velocity of $2 \Delta_{0}$ phonons is smaller than the velocity of sound measured by $B$. Lowering the gap by a magnetic field, as in $J(H)$, causes a shift of the heat pulse towards shorter times. On the other hand, the $L$ bolometer signal reveals a rather peculiar structure in that it reflects the composition of the transmitted $L$ spectrum. The former peak is ascribable to low frequencies $\left(q<q_{\mathrm{TF}}\right)$ since it has no equivalent in the junction signals, while the following hump would correspond to the phonons $\left(q>2 k_{\mathrm{F}}\right)$ of much higher frequency ( $>830 \mathrm{GHz}$ ), and of smaller velocity. The delay expected for the latter due to dispersion is of the right order of magnitude $(\simeq 5 \%$ of the transit time). The stability in arrival time of the junction signal $J(H)$ as the gap is moved is a further indication that the $L$ spectrum contains a void due to electronic absorption. More thorough discussion of these curves will not be pursued in this work (for complements, see ref. [5]).

6. « $2 k_{F}$ " spectroscopy of a heat pulse. -6.1 According to the principle introduced in section 1, the gradual attenuation of a heat pulse with increasing carrier concentration realizes an absorption analysis of its power spectrum. Only the $L$ heat pulse can be studied this way, since $T$ phonons are not coupled to the electrons (cf. eq. (5.1)). The cut-off energy in a sample is defined by the value of $2 \hbar k_{\mathrm{F}} s$ with $k_{\mathrm{F}}=\left(3 \pi^{2} n\right)^{1 / 3}$; its numerical relation to $n$ is shown in table III (the velocity of an $L$ mode propagating along [111] has been taken equal to $3.92 \mathrm{~km} / \mathrm{s}$ ).

The attenuation experiments were performed on a series of samples whose concentrations, as obtained by Hall effect measurements, ranged from $1.9 \times 10^{14}$

\section{TABLE III}

Correspondence of the electron concentration $n$ to the cut-off wavevector $2 k_{\mathrm{F}}$ and cut-off energy $2 \hbar k_{\mathrm{F}} s$, expressed in kelvins $(1 \mathrm{~K} \infty 20.7 \mathrm{GHz})$.

$\begin{array}{ccc}n / \mathrm{cm}^{-3} & 2 k_{\mathrm{F}} / 10^{6} \mathrm{~cm}^{-1} & 2 \hbar k_{\mathrm{F}} s k_{\mathrm{B}}^{-1} / K \\ - & - & 0.85 \\ 10^{14} & 0.29 & 1.85 \\ 10^{15} & 0.62 & 4.0 \\ 10^{16} & 1.33 & 8.5 \\ 10^{17} & 2.87 & 18.5 \\ 10^{18} & 6.19 & 40 \\ 10^{19} & 13.33 & \end{array}$


to $2.0 \times 10^{18} \mathrm{~cm}^{-3}$. A typical set of bolometer signals was shown in figure 4 . The samples had similar lengths, between 6.0 and $9.2 \mathrm{~mm}$; this is necessary because it is observed that the ratio $L / T$ is a slightly varying function of the length in pure samples (intrinsic diffusion has not the same result on the $L$ and $T$ heat pulses).

An absolute attenuation measurement along the lines described in paragraph 5.1 would be too laborious to apply on a number of samples. But, both on theoretical and experimental grounds, it can be taken as granted that the transmission of the $T$ phonons is not affected by the doping. Thus, the $L$ pulse behaviour will be estimated from the variation of the $L$ over $T$ ratio. As an example, figure 8 is a plot as a function of $2 k_{\mathrm{F}}$ of the $L / T$ peak amplitudes obtained for a power incident on the emitter of $0.6 \mathrm{~W}$ or $60 \mathrm{~W} / \mathrm{cm}^{2}$, and a pulse duration of $10^{-7} \mathrm{~s}$. The horizontal bars representative of each sample have the width which corresponds to the extreme values of $n$ measured in several parts of the primitive crystal (the typical concentration inhomogeneity over one centimeter is $<20 \%$, but in one case, rather exceptional, and not very useful for our purposes). Among the samples investigated, one was discarded because it gave inconsistent, still not understood, results. Under this restriction, a smooth curve can be traced through all the experimental results.

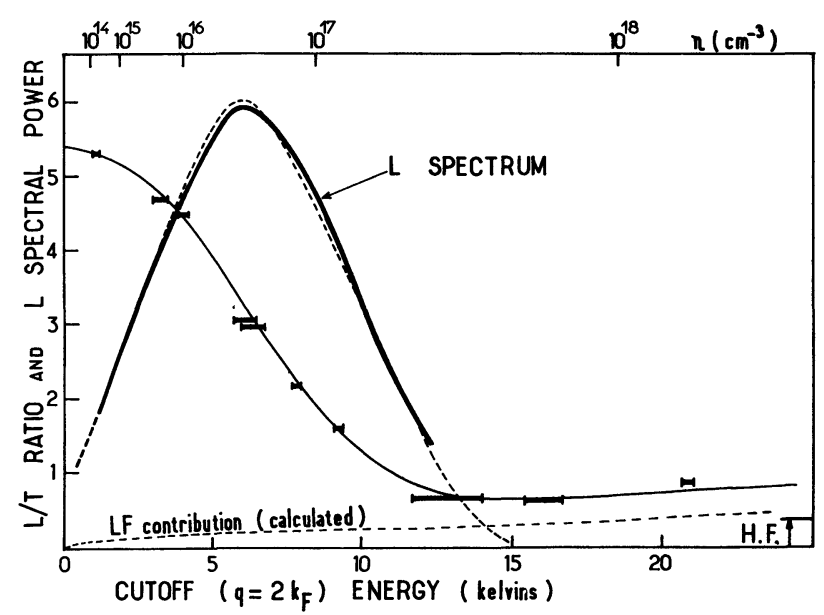

FIG. 8. - Heavy bars : experimental $L / T$ ratios vs. $2 k_{\mathrm{F}}$ (in units of equivalent phonon energy). The continuous line through these bars was computed, with screening effects included, assuming the spectral distribution shown as a dashed line. Heavy curve : $L$ spectrum deduced by simple derivation of $L / T$, according to eq. (6.3).

The main feature of the $L / T$ behaviour is the gradual decrease accompanying the enlarging of the Fermi sphere and to the utilization of which we will come back to soon. Next, above a certain concentration of about 2 to $3 \times 10^{17} \mathrm{~cm}^{-3}$, the $L$ pulse reappears stronger. This upward bending, which was not observed with the superconducting tin diode (Fig. 6a), is readily attributed to the reappearance of the lowfrequency modes and can be accounted for by the screening effects. We remember from figure $1 a$ that $L$ phonons in the range $0-3 \mathrm{~K}$ have free paths which increase with the electron concentration and attain several millimeters when $q_{\mathrm{TF}} \gg q$. Indeed, the contribution of these low-frequency (L.F.) modes to $L / T$ (dashed curve in Fig. 8) is seen to be in order of magnitude agreement with the experiment (for this calculation, the distribution function $P_{\mathrm{L}}(q)$ derived in the next paragraph was assumed).

A special mention is deserved by the heavily doped sample $\left(n=1.0 \times 10^{19} \mathrm{~cm}^{-3}\right)$ which was presented in paragraph 5.3 showing important screening effects. It is not represented here; its $L / T$ ratio would produce the data of figure 8 very far in $2 k_{\mathrm{F}}$ space, consistently with the calculated screening effect on L.F. waves. However, this sample had to be cut to a shorter length $(2.1 \mathrm{~mm})$ than the others because, for the same length, the heat pulses were observed to be wider more diffuse. Furthermore, at this doping, the concentration of electrons might well be in the range where the partial P-nature of the conduction states creates a possibility of scattering of $T$ waves (see $\S 2.1$ in fine). Therefore, this sample will not serve any further for spectrum determination.

\section{2 DeRIVATION OF THE $L$ POWER SPECTRUM. -} Let $P_{\mathrm{L}}(q)$ be the power spectrum incident on the detector in the absence of electrons. Two separate contributions add up to the transmitted signal in a doped crystal. These are the high frequency part :

$$
\int_{2 k_{\mathrm{F}}}^{\infty} P_{\mathrm{L}}(q) \mathrm{d} q
$$

and the low-frequency (LF) part :

$$
\int_{0}^{q_{\mathrm{TF}}} P_{\mathrm{L}}(q) \mathrm{e}^{-l / \Lambda_{\mathrm{s}}(q)} \mathrm{d} q
$$

where $l$ is the sample length and $\Lambda_{\mathrm{s}}$ is the mean free path for the screened electron-phonon interaction given by eq. (2.12). In eq. (6.2), the screening parameter $q_{\mathrm{TF}}\left(=0.62 \times 10^{6} \mathrm{~cm}^{-1}\right.$ for $\left.n=10^{17} \mathrm{~cm}^{-3}\right)$ is used as a practical upper limit of integration (let us recall that $q_{\mathrm{TF}}<2 k_{\mathrm{F}}$ ).

It will be assumed throughout that $P_{\mathrm{L}}(q)$, and similarly the spectrum of transverse phonons, are the same from sample to sample or, in other words, that the dopant impurities introduce no additional scattering by themselves. This assumption should not be understood as an ad hoc simplification of the problem. Rather it is based on the analysis of paragraph 5.1, which showed the transmission of $T$ phonons to be independent of the doping up to $5 \times 10^{17} \mathrm{~cm}^{-3}$ at least. On the other hand, it is generally accepted that, at a given frequency, $L$ waves are not more sensitive to defect scattering than $T$ waves.

Concerning the LF contribution to the transmitted $L$ pulse, we note from figure 8 that the $L$ bolometer 
signal decreases as low as $10 \%$ of the original value in a pure sample, which binds the LF contribution to less than those $10 \%$. It will then be reasonable in a first step to consider the contribution (6.1) alone, with neglect of the LF phonons. The contribution (6.2) will be later introduced as a correction.

The spectral density $P_{\mathrm{L}}(q)$ is proportional to the decremental variation of the $L$ total power between two adjacent frequencies. In practice, according to eq. (6.1), it is given by the derivative :

$$
P_{\mathrm{L}}(q) \propto-\left.\frac{\mathrm{d}(L / T)}{\mathrm{d}\left(2 k_{\mathrm{F}}\right)}\right|_{2 k_{\mathrm{F}}=q} .
$$

The result of such a differentiation is represented as a heavy line in figure 8 . No procedure more sophisticated than a graphical determination of the slopes was applied, thanks to the good fitting of the experimental data to a smooth curve.

Starting with this first approximation, a numerical method including eq. (6.1) and (6.2) was developed by using a programmable calculator. Trial forms of $P_{\mathrm{L}}(q)$ were entered as data and adjusted so as to give for the transmitted signal the best fit to the experimental points. Thus, choosing the spectral distribution shown as a dashed line leads for the transmitted $L$ signal to a function which, indeed, goes nicely to the $L / T$ data (continuous line). Furthermore, this result is rather safe, for it is fairly sensitive to the shape of the trial distribution.

Apart from providing more accuracy on the wings of the $L$ spectrum, this procedure brings only minor improvement to the much simpler differentiation method described earlier, so that the latter will be preferred in the following. The main advantage of neglecting the LF contribution is to make the electron filter depend only upon $k_{\mathrm{F}}$, which is a universal function of the electron concentration.

To obtain the fit between the theory and the experimental $L / T$ data (Fig. 8), the calculated transmission curve (continuous line) was lifted by a constant quantity, symbolized by HF in the right corner of the figure, which we attribute to a background of scattered high-frequency phonons ( $>20 \mathrm{~K})$. The main defence for this assumption - which does not affect the validity of the differentiation method - rests in its smallness $(\sim 7 \%$ of the $L$ signal in the undoped crystal). It is also compatible with other evidences as will be discussed in paragraph 7.1.

6.3 DEPENDENCE UPON POWER AND DURATION OF THE INPUT PULSE. - We have determined the spectral distribution of the $L$ heat pulse for several input powers $\dot{Q}$ in the range 10 to $500 \mathrm{~W} / \mathrm{cm}^{2}$ applied to the emitter gold film. The results are reported in the log$\log$ coordinates of figure 9 where, for each $\dot{Q}$, the proportionality factor left undetermined by eq. (6.3) was chosen such that the integral spectral power be proportional to the amplitude of the detected $L$ signal in an undoped sample. The latter was in fact observed to follow approximately a $\dot{Q}^{0.7}$ dependence rather than a linear dependence. Let us remember at this stage that losses to the helium bath can play an important role [23, 39] in the transfer of energy out of the metal film : a jump in the detected signal versus $\dot{Q}$ occurs at around $100 \mathrm{~W} / \mathrm{cm}^{2}$ for an input duration of $10^{-7} \mathrm{~s}$ and an helium temperature of $1.25 \mathrm{~K}$, corresponding to the formation of a bubble near the hot film. The $\dot{Q}^{0.7}$ dependence is in fact observed below and above this value. In any event, one usually admits that the helium interface affects the total power emitted into the sample, not the shape of the spectrum.

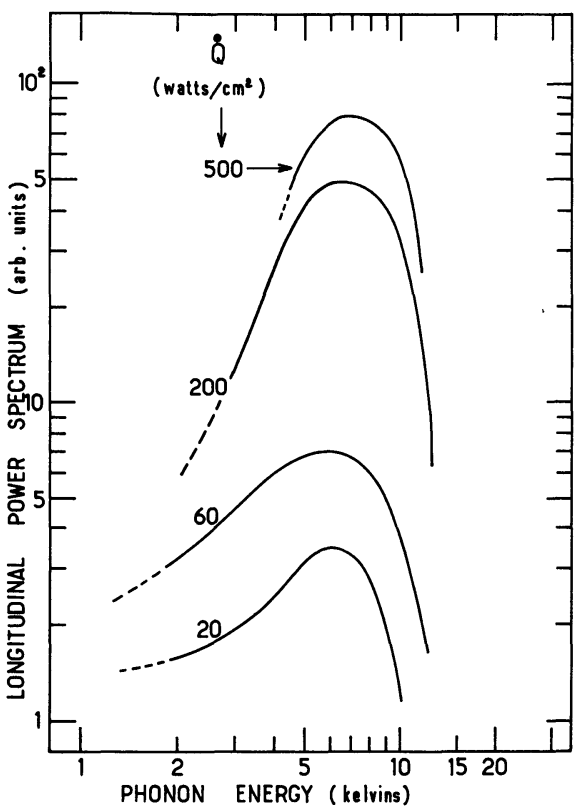

FIG. 9. - Log-log plot of the $L$ frequency spectra for several input powers. Input duration : $100 \mathrm{~ns}$. $T_{0}=1.25 \mathrm{~K}$. The vertical shifts were made to correspond to the amplitudes of the bolometer signals in an undoped crystal.

The effect of varying the input-pulse duration was also considered, although not in detail. Since the $L$ signal saturates with a characteristic time ( $\sim 80 \mathrm{~ns})$ shorter than for the $T$ signal $(\sim 200 \mathrm{~ns})$, the $L / T$ amplitude ratio is a decreasing function of the input duration, and this is a general result for all materials. However, the measured spectral distribution was found weakly duration dependent. For instance, increasing the pulse duration from 100 to $400 \mathrm{~ns}$ at constant power $\left(60 \mathrm{~W} / \mathrm{cm}^{2}\right)$ has upon the $L$ spectrum more or less the same consequences as changing the input power from 60 to $200 \mathrm{~W} / \mathrm{cm}^{2}$ for $100 \mathrm{~ns}$ pulses.

\subsection{DEPENDENCE UPON THE SAMPLE TEMPERATURE.} - Raising the ambient temperature $T_{0}$ results in more profound effects. The $L / T$ amplitude ratios taken at $T_{0}=3.7 \mathrm{~K}$, the maximum temperature compatible with the use of superconducting tin bolometers, are significantly smaller than at lower temperatures for the same conditions of emission 
(Fig. 10). On the other hand, the $L$ spectrum deduced from these data (full curve) differs markedly from the spectrum at $T_{0}=1.25 \mathrm{~K}$ (dashed curve) in that the maximum is shifted to higher energies $(\sim 9 \mathrm{~K})$ and low frequencies are almost absent, as indicated by the strict constancy of $L / T$ at both low and high dopings. The latter fact is comforting for it tells strongly in favour of the introduction of screened low-frequency waves to interpret the data at $T_{0}=1.25 \mathrm{~K}$. On the contrary, here, no reappearance of screened waves is to be expected at high dopings, due to the shape of the spectrum.

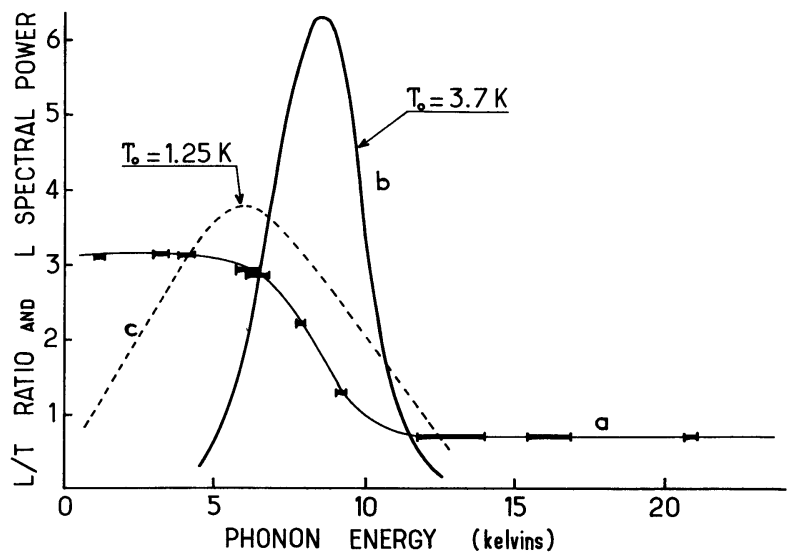

FIG. 10. - (a) $L / T$ ratio measured at $T_{0}=3.7 \mathrm{~K}$ versus $2 h_{\text {I }}$ (in equivalent phonon energy/K). Input power : $60 \mathrm{~W} / \mathrm{cm}^{2}$; duration : 100 ns. (b) Derived $L$ power spectrum. (c) Corresponding spectrum for $T_{0}=1.25 \mathrm{~K}$, normalized to have an area equal to $(b)$.

Finally, the constant background of HF phonons in the $L$ pulse is higher than at $T_{0}=1.25 \mathrm{~K}$, and the heat signals were also wider. These observations are consistent with the idea of some temperature-dependent intrinsic scattering, most likely phonon-phonon scattering (the Debye temperature of $\mathrm{InSb}$ is only $130 \mathrm{~K})$.

One might question the sharpness of the Fermisurface cut-off at non-zero temperatures since the thermal broadening of the Fermi electrons is comparable to the phonon energies of interest. But, in fact, since the phonon energy is linear in $q$, and, with due account of the condition (1.1), rather than the spread in energy, one has to consider the spread in wavevectors. In relative terms, it is given by :

$$
\frac{\Delta\left(2 k_{\mathrm{F}}\right)}{2 k_{\mathrm{F}}}=\frac{1}{2} \times \frac{\Delta \varepsilon_{\mathrm{F}}}{\varepsilon_{\mathrm{F}}} \simeq \frac{T_{0}}{T_{\mathrm{F}}}=10^{-3} \text { to } 10^{-2}
$$

where $T_{\mathrm{F}}=\varepsilon_{\mathrm{F}} / k_{\mathrm{B}}$ is the Fermi temperature and the uncertainty $\Delta$ is related to thermal effects only. Thus, it appears that the $2 k_{\mathrm{F}}$ cut-off remains well defined in the whole liquid-helium temperature range.

6.5 THIN-FILM RESONANCES. - The analysis of phonon spectra was thus far performed assuming throughout a flat response (vs. frequency) of the bolometers used for the detection. While it is conve- nient to ignore the complicated effects which may arise at the solid-solid and solid-helium interfaces, the phonon detection by metal films is susceptible of a more critical analysis. For the Sn thin-films we described in paragraph 3.2 , the thickness (2000 $\AA$ ) is larger than the mean free path of thermal phonons ( $1000 \AA)$ in normal tin [40], and therefore no frequency discrimination is imparted by bolometric detection.

This conclusion would certainly not hold for much thinner films or for materials having a weaker electronphonon coupling. We undertook the same set of experiments as described in paragraph 6.1 by using pure aluminum bolometers (thickness : $370 \AA$ ) in which the absorption length is $5000 \AA$ (for $5 \mathrm{~K}$ phonons) according to transmission measurements [41]. First of all, the ratio $L / T$ of longitudinal to transverse peak amplitudes (see Fig. 11, lower curve) was found to be about twice as small as that measured with a tin bolometer, and this points to the relativity of even such direct measurements as the ratio of different polarizations (another value was indeed obtained by using a lead bolometer).

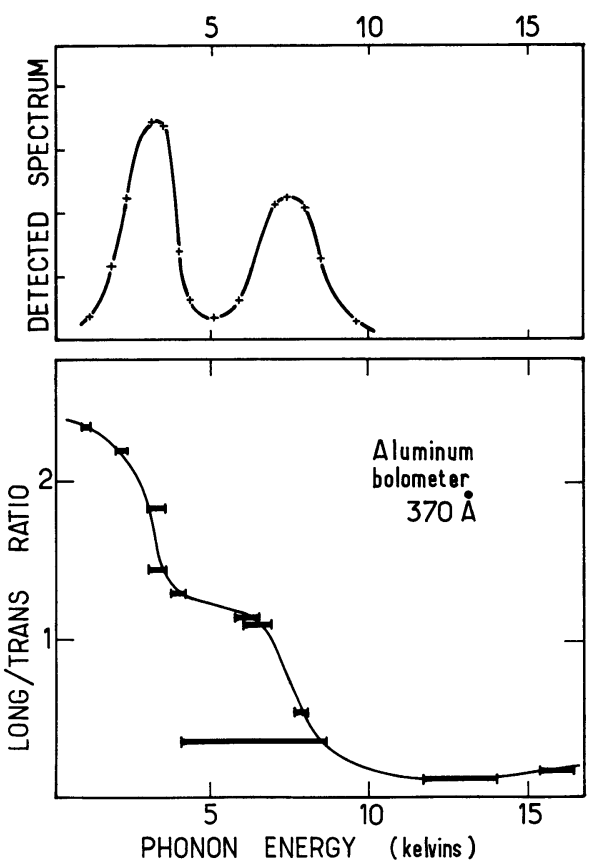

FIG. 11. - Lower graph : $L / T$ ratio measured with an aluminum bolometer at $T_{0}=1.25 \mathrm{~K}$. Power : $60 \mathrm{~W} / \mathrm{cm}^{2}$; duration $100 \mathrm{~ns}$. Upper graph : derivative of the lower curve with respect to the phonon energy, showing resonance in the metal film.

Secondly, the detected spectrum, which is the product of the incident energy distribution - recovered in principle with a tin detector - by the response function of aluminum, presents a clear oscillatory structure (Fig. 11, upper curve). The minimum around $5 \mathrm{~K}$ is the correspondent of the accident occurring in the measured amplitude ratio $L / T$ versus $2 k_{\mathrm{F}}$ (lower curve). Experimental data in this region were taken with special care and their repeatability checked 
since the interpretation relies strongly on their accuracy. This structure can be understood as an acoustic resonance phenomenon such as already reported [42], taking place inside the aluminum bolometer film. If $d$ is the film thickness, the interval $\Delta q$ between two maxima of absorption reads :

$$
\Delta q=\frac{\pi}{d}=8.5 \times 10^{5} \mathrm{~cm}^{-1}
$$

or an equivalent energy of $\sim 2.0 \mathrm{~K}$ for a longitudinal phonon of velocity $6.5 \mathrm{~km} / \mathrm{s}$ propagating in aluminum. The observed spacings agree correctly with this estimate which, however, the limitation of the phonon spectrum transmitted by InSb did not allow to check at higher orders of interference. Also significant here is the contrast in the fringe pattern. It is not excluded that similar interference effects exist in the emitter film, although no evidence for that has yet been reported.

7. Discussion of the results. -7.1 COMPARISON TO THE THEORETICAL MODELS. - The spectral distributions of heat pulses derived in the preceding section (see Fig. 9) are characterized, whatever the input power, by a maximum around $7 \mathrm{~K}$, equivalent to a pulse temperature of about $2 \mathrm{~K}$, and a rather rapid drop above $12 \mathrm{~K}$. These numbers are well below those predicted by any model of Joule emission. If we were to reconcile our results with theory, we should allow for a strong alteration of the heat pulse in the course of its propagation through the samples, for which the scattering of high-frequency modes by lattice defects (isotopes) provides the most likely explanation.

With this in mind, we plotted in figure 12 the experimentally derived spectra $P_{\mathrm{L}}(q)$ at $T_{0}=1.25 \mathrm{~K}$ in front of the Planck spectra (dotted lines) corresponding to the same input powers. The pulse tempe-

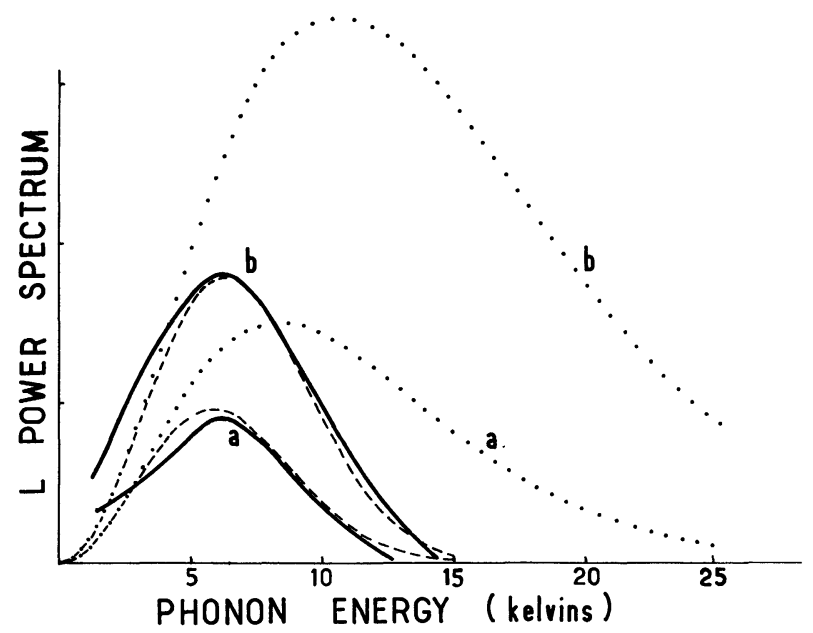

FIG. 12. - Comparison of the experimental spectra (-) with the blackbody spectra $(. . .$.$) , corrected for volume scattering (--.-).$ $T_{0}=1.25 \mathrm{~K}$; pulse duration : $100 \mathrm{~ns}$. The set of curves $(a)$ corresponds to a power of $20 \mathrm{~W} / \mathrm{cm}^{2}\left(T_{\mathrm{em}}=2.8 \mathrm{~K}\right)$, while the $(b)$ set corresponds to $60 \mathrm{~W} / \mathrm{cm}^{2}\left(T_{\mathrm{em}}=3.7 \mathrm{~K}\right)$. ratures were estimated by assuming perfect acoustic match for simplicity $(\eta=1$ in eq. (3.2)). These spectra can only be plotted within a multiplication factor since the sensitivity of the bolometers is not absolutely calibrated. Therefore, this factor, - the same for the different powers - was chosen such that the Planck spectra fit correctly the experimental results at low frequencies. The disagreement at higher frequencies is patent. Then, an intrinsic - independent of the doping - mean free path of the form :

$$
\Lambda_{\mathrm{i}}(\mathrm{cm})=\left(\frac{q_{\mathrm{i}}}{q}\right)^{\alpha} \times(1 \mathrm{~cm})
$$

was imposed upon the blackbody spectra and a fit to $P_{\mathrm{L}}(q)$ was searched by varying the parameters $\alpha$ and $q_{\mathrm{i}}$. The dashed curves of figure 12 thus result from the choice :

$$
\left.\begin{array}{rl}
\alpha & =2.8 \\
q_{\mathrm{i}} & =3.0 \times 10^{6} \mathrm{~cm}^{-1}
\end{array}\right\} .
$$

Consideration of the experimental spectra at either different input powers or at a different lattice temperature $\left(T_{0}=3.7 \mathrm{~K}\right)$ leads to only small deviations $(\alpha=2.8 \pm 0.2)$ around these values, which may therefore be regarded as well determined. The other way round, the high-frequency attenuation is so strong that the present best-fitting procedure will not provide a sensitive test of the generated spectrum itself, and cannot serve the purpose of discriminating between the different theories of Joule emission.

From thermal conductivity measurements as a function of temperature [22], the Rayleigh $(\alpha=4)$ scattering was characterized by a wavevector $q_{\mathrm{i}}=7.4 \times 10^{6} \mathrm{~cm}^{-1}$, leading to an attenuation weaker than implied by eq. (7.2). However, both determinations should not be strictly compared since such processes as phonon-phonon collisions were substracted from the thermal resistivity to yield the Rayleigh scattering, whereas eq. (7.2) is an overall estimate. Another point is that thermal conductivity refers to a steady state of quasi-equilibrium (small temperature gradients), different from the heatpulse situation.

We shall now attempt to interpret some gross experimental facts within the framework of an effective pulse temperature model plus an intrinsic scattering of the type represented by eq. (7.1).

- The measured temperature excursion of the bolometer under a given phonon radiation is about one hundred times smaller than expected from the specific heat content and relaxation time $(\sim 20 \mathrm{~ns})$ of the bolometer film. The difference between the blackbody spectrum and the transmitted spectrum (dashed curve in Fig. 12) accounts for part of this observation. The rest may be found in the efficiency of the bolometer itself, and in losses to the helium bath. 
- The less-than-linear dependence $(\S 6.3)$ of the bolometer signal upon the input power results from the shift of the phonon spectrum towards high frequencies.

- The rather slow rises of the $L$ and $T$ signals ( $\$ 6.3$ ), the slower the higher the input power, are not predicted by any emission theory. On the contrary, in the present description, the slow rises reflect the arrival of the phonons having been scattered under low angle, not to mention the dispersion effects.

- A strong back-scattering of the HF phonons could explain the surprisingly long (a few $10^{-8} \mathrm{~s}$ ) thermal relaxation times of the normal metallic films [43].

Finally, one cannot gloss over another possible interpretation suggested by the relative insensitivity of the experimental spectra upon the input power. It is well established from Raman scattering studies [44] that mechanical polishing of the samples causes surface damage as deep as several microns; the evaporated $\mathrm{SiO}$ layer used for insulation is not either a perfect crystal. In the extreme hypothesis, this would produce a global heating of the crystal just behind the phonon generator and result in an effective pulse temperature close to $T_{0}$, approximately consistent with our data at $1.25 \mathrm{~K}$. Comparable propositions were made for heat pulse propagation in different materials, $\mathrm{MgO}$ [45] and Ge [46]. The feeling of the present authors is that so severe a down-conversion of the phonon frequencies is not a necessary assumption, except in dirty materials or at very high phonon intensities. We notice that the high frequency cut-off of our spectra does not follow the variation of the lattice temperature (Fig. 10). In fact, many observations made with tunnel junctions, including chromatic-dispersion measurements [38], contradict the idea of a surface thermalization. Let us remark also that, explained on this basis, the slow risetimes of the $L$ and $T$ heat-pulse signals should be equal, which is contrary to experimental evidence.

7.2 MEasurement OF NON-ELECTRONIC VOLUME SCATTERING. - To our knowledge, the only spectral analysis of a superthermal phonon population, as a function of the distance from the source, was per-, formed by means of the spin-phonon spectrometer [8]. But, because (i) this examination was done in the steady regime and (ii) the scattering of the phonons took place at the solid-helium boundary, the results of this experiment are not quite relevant to the common situation. We now describe preliminary measurements of the overall volume attenuation of heat pulses in indium antimonide. No $2 k_{\mathrm{F}}$ spectroscopy being intended at this stage, only undoped material was considered.

A pure InSb crystal, oriented along the [111] direction, was cut into three chips of lengths 2.3, 4.6 and $9.5 \mathrm{~mm}$. The samples were prepared simultaneously and mounted in a single sample-holder.
The three bolometers were calibrated against each other by second sound pulses in the liquid helium bath $\left(T_{0}=1.25 \mathrm{~K}\right)$, according to the procedure introduced in paragraph 5.1. Equal electrical pulses were then applied to the three emitters. Figure 13 is a semi-log plot, versus the sample length, and for several conditions of emission, of the intensity of the phonon beam. The latter was deduced, within a

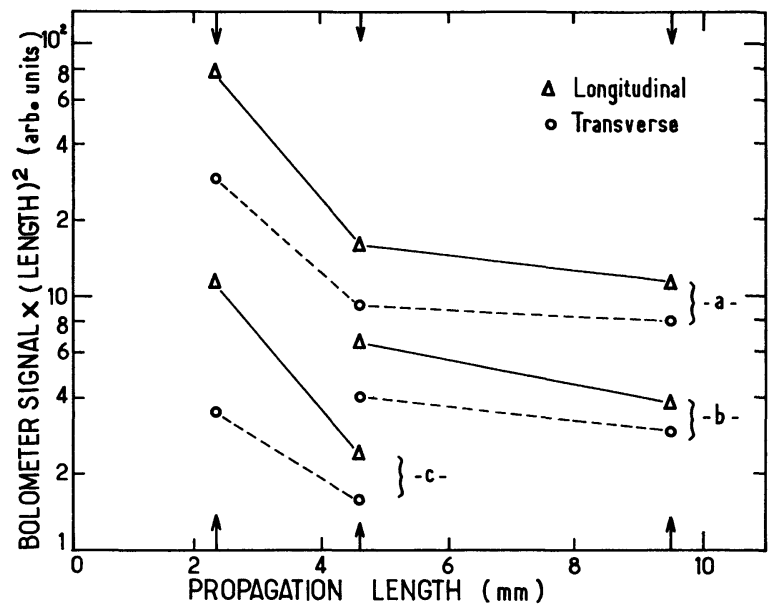

FIG. 13. $-L$ and $T$ phonon signal amplitudes, normalized for equa solid angles, plotted as a function of the length of propagation (3 values), for several input powers and durations. (a) $: 80 \mathrm{~W} / \mathrm{cm}^{2}$, $200 \mathrm{~ns} ;(b): 50 \mathrm{~W} / \mathrm{cm}^{2}, 100 \mathrm{~ns} ;(c): 14 \mathrm{~W} / \mathrm{cm}^{2}, 100 \mathrm{~ns}$. Comparison is relevant only between data linked by a stroke.

constant factor, by making the product (bolometer signal) $\times$ (sample length $)^{2}$, in order to account for the difference in solid angles (possible differential self-focusing effects were ignored). The behaviour of the beam intensity is a measure of volume scattering : in a semi-log plot, a non-scattered beam should be represented by an horizontal line, while a homogeneous decay would be characterized by a straight line with negative slope. The results for both the $L$ and $T$ modes indicate that the composition of the ballistic beam is changing during the flight. The attenuation is especially important in the first millimeters of the propagation, where the heat pulse is rich in high-frequency phonons. The heat pulses transmitted along about $8 \mathrm{~mm}$ were shown $(\S 6.2)$ not to appreciably contain energies above $15 \mathrm{~K}$. But, on the other hand, one has at least two evidences that denote the presence of HF phonons near the generator :

a) the $L$ signal for the heavily-doped sample presented in figure 7 consists mainly of energies larger than $40 \mathrm{~K}$ having travelled $2.14 \mathrm{~mm}$;

b) $L$ and $T$ heat pulses could be detected [38] by lead-thallium junctions with $2 \Delta=2.25 \mathrm{meV} \sim 26 \mathrm{~K}$ on samples $2 \mathrm{~mm}$ thick, while they were barely visible for larger thicknesses.

To summarize, the heat pulses transmitted by pure InSb are shaped by intrinsic scattering mechanisms, such as Rayleigh scattering by isotopes and phonon- 
phonon collisions. In order to trace back to the Joule generated distribution, the signals should be probed rather close to the emitter $(\sim 1 \mathrm{~mm})$.

8. Conclusion. - The heat pulse method, whose capabilities are increased by the use of magneticallytunable superconducting diodes as quantum detectors, is a valuable tool for monitoring the transport of ballistic phonons. It permits one to select a wavevector direction and, thanks to time-of-flight discrimination, to separate the different polarizations and, to a lesser extent, the different frequencies. The elastic constants of indium antimonide could thus be measured within about $0.5 \%$ accuracy (eq. 4.3) and the dispersion relations be determined at long wavelengths (ref. [38]). In the same way, some familiar properties of the electron-phonon interaction : coupling of the longitudinal phonons to the deformation potential, non-interaction of the transverse phonons with a simple conduction band (S-symmetry), cut-off at $q=2 k_{\mathrm{F}}$, and screening effects, were shown in a transparent manner (section 5).

Absorption by conduction electrons up to $q=2 k_{\mathrm{F}}$ realizes an unequalled phonon filter as far as the definition of the edge is concerned (which, paradoxically, prevents the measurement of the strength, or deformation potential, of the electron-phonon interaction). This tunable filter allowed a true spectral analysis, in the range of 20 to several hundreds of $\mathrm{GHz}$, of the heat generated by Joule effect in a metal film and transmitted through InSb (extension to less broadband sources is in progress). It was found that the concept of an effective pulse temperature correlates qualitatively most of the experimental facts, without resorting to a mechanism of surface thermalization. A new value for the mean free path limited by intrinsic scattering was proposed (eq. 7.2). However, due to the poor transmissivity of InSb at high frequencies, the problem of Joule generation of phonons cannot be considered as clarified.

Acknowledgments. - Original impulse and interest in this subject belong to Albert Zylbersztejn, from Thomson-C.S.F. Drs Besson and Morand, from the Société Anonyme des Télécommunications, provided most of the InSb samples. Discussions with Mrs. N. Perrin, J. Vannimenus are also sincerely acknowledged.

\section{References}

[1] Von Gutfeld, R. J., in Physical Acoustics, edited by W.P. Mason (Academic Press, New York, 1968). Vol. V, p. 233

[2] J. Physique Colloq. 33 (1972) C4. La Physique des Phonons de Très Haute Fréquence.

[3] Maneval, J. P., Zylbersztejn, A. and Huet, D., Phys. Rev. Lett. 27 (1971) 1375.

[4] Carlson, D. G. and Segmuller, A., Phys. Rev. Lett. 27 (1971) 195 .

[5] Huet, D. and Maneval, J. P., Phys. Rev. Lett. 33 (1974) 1154.

[6] Narayanamurti, V., Phys. Lett. 30A (1969) 521.

[7] Wigmore, J. K., Phys. Rev. B 5 (1972) 700.

[8] Anderson, C. H. and Sabisky, E. S., in Physical Acoustics, edited by W. P. Mason (Academic, New York) 1971, Vol. VIII.

[9] Semiconductors and Semimetals, edited by R. K. Willardson and A. C. Beer (Academic, New York) 1966, Vol. I and II.

[10] Korenblit, L. L. and Sherstobitov, V. E., Sov. Phys. Semicond. 2 (1968) 564

[11] Bardeen, J. and Shockley, W., Phys. Rev. 80 (1950) 72.

[12] Hunter, S. C. and Nabarro, F. R. N., Proc. R. Soc. A 220 (1953) 542.

[13] Meijer, H. J. G. and Polder, D., Physica 19 (1953) 255.

[14] Zawadski, W. and Boguslawski, P., Phys. Rev. Lett. 31 (1973) 1403.

[15] Ziman, J. M., Phil. Mag. 1 (1956) 191 and Corrigendum, ibid. p. 292.

[16] Szymańska, W. and Maneval, J. P., Solid State Commun. 8 (1970) 879;

See also : Maneval, J. P., thèse d'Etat, Paris (1973) unpublished.

[17] Woll, E. J., Jr. and Kohn, W., Phys. Rev. 126 (1962) 1693.

[18] Bardeen, J., Phys. Rev. 52 (1937) 688.

[19] ARLT, G. and QuAdFlieg, P., Phys. Status Solidi 25 (1968) 323 ; See also : Arnaud, A. and Quentin, G., Phys. Lett. 32A (1970) 16.

[20] Zawadski, W., Adv. Phys. 23 (1974) 435.

[21] Johnson, E. J. and Dickey, D. H., Phys. Rev. B 1 (1970) 2676.

[22] Holland, M. G., Phys. Rev. 134A (1964) 471.
[23] WeIS, O., p. 48 of ref. [2]

[24] Perrin, N. and Budd, H. F., Phys. Rev. Lett. 28 (1972) 1701 ; See also MARIs, H. J., p. 3 of ref. [2].

[25] Cheeke, J. D. N., Hebral, B. and Martinon, C., J. Physique 34 (1973) 257.

[26] Eisenmenger, W. and Dayem, A. H., Phys. Rev. Lett. 18 (1967) 125.

[27] Truell, R., Elbaum, C. and Chick, B. B., Ultrasonic Methods in Solid State Physics (Academic, New York) 1969.

[28] Musgrave, M. J. P., Crystal Acoustics (edited by J. J. Brandstatter) 1970.

[29] Potter, R. F., Phys. Rev. 103 (1956) 47.

[30] Slutsky, L. J. and Garland, C. W., Phys. Rev. 113 (1959) 167.

[31] Maris, H. J., J. Acoust. Soc. Am. 50 (1970) 812.

[32] Maneval, J. P. and Huet, D., Phys. Lett. 48A (1974) 463.

[33] Nill, K. W. and McWhorter, A. L., Proc. Int. Conf. Phys. Semiconductors, Kyoto (1966) p. 755

[34] Lindenfeld, P., Phys. Status Solidi 14 (1966) K47.

[35] Shalyt, S. S., Tamarin, P. V. and Ivleva, V. S., Phys. Lett. 32A (1970) 29.

[36] Comès, R., Lambert, M. and Launois, H., Zeller, H. R., Phys. Rev. B 8 (1973) 571.

[37] Crosby, C. R. and Grenier, C. G., Phys. Rev. B 4 (1971) 1258.

[38] Huet, D., Maneval, J. P. and Zylbersztejn, A., Phys. Rev. Lett. 29 (1972) 1092.

[39] Pannetier, B. and Zylbersztejn, A., p. 69 of ref. [2].

[40] Assumed to be comparable to the mean free path of ( $\hbar q s>2 \Delta$ ) phonons in superconducting tin, for which the value $600 \AA$ was proposed by DyNes, R. C. and NarayanaMURTI, V., Phys. Rev. B 6 (1972) 143.

[41] LoNG, A. R., p. 73 of ref. [2].

[42] Kinder, H., Z. Phys. 262 (1973) 295.

[43] Maneval, J. P., unpublished.

[44] Evans, D. J. and Ushioda, S., Phys. Rev. B 9 (1974) 1638

[45] Wigmore, J. K., Phys. Lett. 37A (1971) 293.

[46] Figldly, T. A., Ishiguro, T. and Elbaum, C., Phys. Rev. B 7 (1973) 1392. 